\title{
Changing Microcircuits in the Subplate of the Developing Cortex
}

\author{
Sarada Viswanathan, ${ }^{1}$ Sharba Bandyopadhyay, ${ }^{1}$ Joseph P. Y. Kao, ${ }^{2}$ and Patrick 0. Kanold ${ }^{1}$ \\ ${ }^{1}$ Department of Biology, Institute for Systems Research, and Program in Neuroscience and Cognitive Science, University of Maryland, College Park, \\ Maryland 20742, and ${ }^{2}$ Center for Biomedical Engineering and Technology and Department of Physiology, University of Maryland School of Medicine, \\ Baltimore, Maryland 21201
}

Subplate neurons (SPNs) are a population of neurons in the mammalian cerebral cortex that exist predominantly in the prenatal and early postnatal period. Loss of SPNs prevents the functional maturation of the cerebral cortex. SPNs receive subcortical input from the thalamus and relay this information to the developing cortical plate and thereby can influence cortical activity in a feedforward manner. Little is known about potential feedback projections from the cortical plate to SPNs. Thus, we investigated the spatial distribution of intracortical synaptic inputs to SPNs in vitro in mouse auditory cortex by photostimulation. We find that SPNs fell into two broad classes based on their distinct spatial patterns of synaptic inputs. The first class of SPNs receives inputs from only deep cortical layers, while the second class of SPNs receives inputs from deep as well as superficial layers including layer 4 . We find that superficial cortical inputs to SPNs emerge in the second postnatal week and that SPNs that receive superficial cortical input are located more superficially than those that do not. Our data thus suggest that distinct circuits are present in the subplate and that, while SPNs participate in an early feedforward circuit, they are also involved in a feedback circuit at older ages. Together, our results show that SPNs are tightly integrated into the developing thalamocortical and intracortical circuit. The feedback projections from the cortical plate might enable SPNs to amplify thalamic inputs to SPNs.

\section{Introduction}

Subplate neurons (SPNs) are a largely transient neuronal cell population that reside in the neonatal cortical white matter (Kostovic and Rakic, 1980; Kanold and Luhmann, 2010) and that play a key role in the segregation and functional maturation of thalamocortical and intracortical circuits (Ghosh and Shatz, 1992; Kanold et al., 2003; Kanold and Shatz, 2006). SPNs in primary sensory areas receive thalamic inputs before thalamic axons grow into the overlying cortical plate and provide feedforward excitation to neurons in the cortical plate (Friauf and Shatz, 1991; Finney et al., 1998; Hanganu et al., 2002; Higashi et al., 2002; Molnár et al., 2003; Piñon et al., 2009; Zhao et al., 2009). SPNs extend neurites into the cortical plate and thus might also receive feedback projections from the cortical plate that could influence processing of thalamic inputs in SPNs (Friauf et al., 1990; Friauf and Shatz, 1991; Hanganu et al., 2002; Hanganu and Luhmann, 2004; Piñon et al., 2009; Zhao et al., 2009). Given the key role of

Received Sept. 16, 2011; revised Nov. 2, 2011; accepted Dec. 5, 2011.

Author contributions: S.V., S.B., and P.O.K. designed research; S.V., S.B., and P.O.K. performed research; S.B., J.P.Y.K., and P.O.K. contributed unpublished reagents/analytic tools; S.V., S.B., and P.O.K. analyzed data; S.V., J.P.Y.K., and P.O.K. wrote the paper.

This work was supported by NIH Grant R01 DC009607, the International Cerebral Palsy Research Foundation, National Alliance for Research on Schizophrenia and Depression, and the Alfred P. Sloan Foundation (P.O.K.), and by NIH Grant R01 GM056481 (J.P.Y.K.). We thank Dr. Karel Svoboda (HHMI Janelia Farm Research Campus, Ashburn, VA), Loren Looger (HHMI Janelia Farm Research Campus, Ashburn, VA), and Barak Shechter for technical assistance; Aminah Sheikh, Julie Zemskova, and Justin Penzenstadler for histological assistance; and Dan Winkowski, Krystyna Orzechowski, and Paul Watkins for helpful comments on this manuscript.

Correspondence should be addressed to Patrick 0. Kanold at the above address. E-mail: pkanold@umd.edu. DOI:10.1523/JNEUROSCI.4748-11.2012

Copyright $\odot 2012$ the authors $\quad 0270-6474 / 12 / 321589-13 \$ 15.00 / 0$
SPNs relaying thalamic activity to the cortical plate, we investigated the existence and spatial origins of intracortical projections to SPNs by in vitro recordings and laser-scanning photostimulation (LSPS) (Shepherd et al., 2003) in thalamocortical brain slices from neonatal (P2-P14) mouse primary auditory cortex (A1).

We find that SPNs receive excitatory and inhibitory inputs from the developing cortex. SPNs could be separated into two classes. The first class of SPNs receives cortical inputs that originate solely in deeper cortical layers. The second class of SPNs receives cortical inputs from deep as well as superficial cortical layers, including layer 4, the target of SPN projections (Friauf et al., 1990; Zhao et al., 2009). SPNs showing superficial cortical inputs became more prevalent in the second postnatal week. This indicates either a gradual strengthening in the feedback projections or a selective loss of SPNs that did not receive feedback projections. In addition, we find that, at older ages, SPNs that receive input from the superficial cortical plate are located more superficially than those SPNs that do not.

Together, these results demonstrate the existence of distinct functional subpopulations of SPNs and that the developing cortical circuit can influence SPN function. Thus, both ascending inputs and feedback connection to SPN might contribute to the role of SPNs in cortical development.

\section{Materials and Methods}

Slice physiology methods are as published previously (Zhao et al., 2009). All procedures followed the University of Maryland College Park animal use regulations.

Slice preparation. We use mice (C57BL/6) of either sex from The Jackson Laboratory (jax.org) or mice in which connective tissue growth factor 
(CTGF)-expressing SPNs are labeled with GFP [Tg(Ctgf-EGFP)156Gsat; Mutant Mouse Regional Resource Center] to verify our recording locations by the position of the recording electrode relative to GFP-expressing cells (see Figs. 1, 3). Mice are deeply anesthetized with isoflurane (Halocarbon). A block of brain containing A1 and the medial geniculate nucleus (MGN) is removed and thalamocortical slices $(500-600 \mu \mathrm{m}$ thick) are cut on a vibrating microtome (Leica) in ice-cold ACSF containing the following (in mM): $130 \mathrm{NaCl}, 3 \mathrm{KCl}, 1.25 \mathrm{KH}_{2} \mathrm{PO}_{4}, 20 \mathrm{NaHCO}_{3}, 10$ glucose, 1.3 $\mathrm{MgSO}_{4}, 2.5 \mathrm{CaCl}_{2}, \mathrm{pH} 7.35-7.4$, in $95 \% \mathrm{O}_{2}-5 \% \mathrm{CO}_{2}$. The cutting angle is $\sim 15^{\circ}$ from the horizontal plane (lateral raised) at older ages and somewhat steeper at younger ages to compensate for the lateral growth of cortex (Cruikshank et al., 2002; Zhao et al., 2009). Slices are incubated for $1 \mathrm{~h}$ in ACSF at $30^{\circ} \mathrm{C}$ and then kept at room temperature. For recording, slices are held in a chamber on a fixed-stage microscope (Olympus BX51) and superfused (2-4 $\mathrm{ml} / \mathrm{min}$ ) with ACSF at room temperature to reduce spontaneous activity in the slice. The location of the recording site in A1 was identified by landmarks (Cruikshank et al., 2002; Zhao et al., 2009).

Identification of subplate. The subplate zone is readily identified as a relatively cell-sparse area between the large layer 6 pyramidal cells and the intermediate zone (see Fig. 1). The subplate encompasses horizontally oriented cells in an upper sublamina below layer 6 (future layer $6 \mathrm{~b}$; see Fig. $2 A$, indicated with "u") and deeper, more scattered neurons in the future white matter (see Fig. $2 A$, indicated with "l"). The upper sublamina is separated from layer 6 by a cell-sparse zone. This subcompartmentalization is much more pronounced in primates and humans (Wang et al., 2011). Compared with other neurons, the deeper subplate neurons in young rodents stain relatively poorly for markers such as NeuN but are apparent under infrared illumination or differential interference contrast (DIC) microscopy. Subplate neurons are intermingled with horizontally traveling fibers, and subplate neurons can be readily seen under DIC due to their horizontal appearance (see Figs. 2, 3). In a subset of recordings, we confirmed our recording location by using mice in which subsets of subplate neurons were labeled with GFP (CTGF-GFP mice) (see Figs. 1, 3). For measurement of the soma location of SPNs, we used the relative position between the bottom of layer 6 and the ventricular zone as both were readily identifiable under DIC.

Electrophysiology. Whole-cell recordings are performed with a patchclamp amplifier (Multiclamp 700B; Molecular Devices) using pipettes with input resistance of $4-8 \mathrm{M} \Omega$. Data acquisition is performed by National Instruments AD boards and custom software (Ephus) (Shepherd et al., 2003). Ephus (available at http://www.neuroptikon.org/projects/ display/ephus/Ephus) is written in MATLAB (Mathworks) and adapted to our setup. Voltages were corrected for an estimated junction potential of $10 \mathrm{mV}$. Electrodes are filled with the following (in mM): 115 cesium methanesulfonate $\left(\mathrm{CsCH}_{3} \mathrm{SO}_{3}\right), 5 \mathrm{NaF}, 10$ EGTA, 10 HEPES, $15 \mathrm{CsCl}$, 3.5 MgATP, 3 QX-314, pH 7.25, 300 mOsm. Biocytin or Neurobiotin $(0.5 \%)$ is added to the electrode solution as needed. Series resistances were typically $20-25 \mathrm{M} \Omega$.

Photostimulation. The $0.5-1 \mathrm{~mm}$ caged glutamate ( $\mathrm{Ncm}-\mathrm{Glu}$ [ $\mathrm{N}-(6-$ nitro-7-coumarylmethyl)-L-glutamate]) (Kao, 2006) is added to the ACSF. Without UV light, this compound has no effect on neuronal activity (Kao, 2006). UV laser light (500 mW, $355 \mathrm{~nm}, 100 \mathrm{kHz}$ repetition rate; DPSS; $0.5-5$ ms pulses) is split by a $33 \%$ beam splitter (CVI Melles Griot), attenuated and shuttered by a Pockels Cells (Conoptics) and shutter (nmLaser), and coupled into a microscope via scan mirrors (Cambridge Technology) and a dichroic mirror (Shepherd et al., 2003). The laser beam in LSPS enters the slice axially through the objective (Olympus; $10 \times, 0.3 \mathrm{NA} /$ water) and has a diameter of $<20 \mu \mathrm{m}$. Laser power at the sample is $<25 \mathrm{~mW}$. We varied laser power and determined that this power provided reliable activation of neurons over all ages (Zhao et al., 2009) (also see Fig. 2). The same settings were used over all ages. We typically stimulated up to $30 \times 30$ sites spaced $20-50 \mu \mathrm{m}$ apart, enabling us to probe areas of $1 \mathrm{~mm}^{2}$. Stimuli are applied at $0.5-1 \mathrm{~Hz}$. Analysis was performed essentially as described previously (Shepherd et al., 2003; Zhao et al., 2009) with custom software written in MATLAB. Activation profiles of layer 4 and layer 5/6 neurons were produced by recording in cell-attached mode while mapping the same region and recording action potentials. To detect monosynaptically evoked PSCs, we detected peak PSC amplitudes in a $\sim 50 \mathrm{~ms}$ time window after the stimulation. We measured both peak amplitude and transferred charge. Transferred charge was measured by integrating the PSC. Traces containing a short-latency ( $<10-15 \mathrm{~ms}$ "direct") response were discarded from the analysis (see Figs. 2, 3, black patches in color-coded maps) as were traces that contained longer latency inward currents of long duration $(>100 \mathrm{~ms})$. These currents could sometimes be seen in locations surrounding $(<50 \mu \mathrm{m})$ areas that gave a direct response. Occasionally, some of the direct responses contained synaptically evoked responses that we did not separate out, leading to an underestimation of local short-range connections. Cells that did not show any large $(>100 \mathrm{pA})$ direct responses were excluded from the analysis as these could reflect astrocytes or migrating neurons. It is likely that the observed PSCs at each stimulus location represent the activity of multiple presynaptic cells. To measure the spatial distribution of inputs, we computed the "laminar" (depth) and "columnar" (perpendicular to laminar) response profiles (Shepherd et al., 2005). We first measured the peak PSC (EPSC or IPSC) amplitude (or PSC charge) and plotted it as function of laser position. We computed values between neighboring stimulus positions by bilinear interpolation (see Fig. 3). For laminar response profiles, the PSCs parallel to the pia were summed for every depth position (see Figs. 3, 6). Layer boundaries were determined from the DIC pictures and confirmed by comparison with the expression of lamina specific markers (L4: ROR-a; SP: CTGF) obtained from the Allen Brain Atlas at P4 and P14 (http:// developingmouse.brain-map.org) or from direct visualization of SCCN1a, which is expressed in layer 4 and in thalamus and thus selectively labels layer 4. We used a transgenic mouse expressing cre recombinase under the control of Scnnla promoter [B6;C3- $\mathrm{Tg}(\mathrm{Scnn} 1 \mathrm{a}-\mathrm{cre}) 3 \mathrm{Aibs} / \mathrm{J}$; JAX strain 9613], crossed them with a mouse that expresses td Tomato in a cre-dependent manner [B6.Cg-Gt(ROSA)26Sor ${ }^{\text {tm9(CAG-tdTomato }) H z e}$; Jax strain 7909]. We used a thalamocortical slice preparation from the resultant offspring to look at the red fluorescence in layer 4 (see Fig. $4 B$ ). Then the laminar response profiles in each layer were summed resulting in the total response originating in each layer (total amplitude or total charge). The fractional input originating from each layer was calculated by dividing the total response in each layer by the summed total response from all layers. The profile peaked around the cell body and fell off at larger distances. The total extent of laminar input was calculated as the width at $20 \%$ of the peak amplitude. For columnar response profiles, PSCs at every depth tangential to the pia were summed for every tangential position. We then measured the $20 \%$ width of the peak amplitude (see Fig. 6). To probe for different classes of SPNs, we used $k$-means clustering to minimize the total absolute distance (MATLAB 7.11; Statistics Toolbox 7.4). We clustered on either the excitatory or inhibitory fractional charge (or amplitude), or used both the fractional excitatory and inhibitory input. To test the validity of clustering and identify how many cell classes were present, we increased the numbers of clusters from 1 until 10 and calculated the fraction of variance that the clusters explained ( $R$-squared) or the Dunn index.

Two-photon photostimulation and two-photon imaging. Two-photon photostimulation and two-photon imaging were performed on a Prairie IV microscope outfitted with a MaiTai Deep See and a Coherent Ultra II femtosecond laser. Slices were incubated in $10 \mu \mathrm{M}$ Oregon Green 488 BAPTA-1 acetoxymethyl ester (OGB-1 AM), washed, and then for stimulation and imaging bathed in $3 \mathrm{~mm}$ MNI-glutamate (Tocris) and imaged at $840 \mathrm{~nm}$. Photostimulation was performed at $725 \mathrm{~nm}$ by sequentially stimulating spots around the cell body of the target cell (1 ms each with a gap of $1 \mathrm{~ms}$ ). Ten spots were selected manually around targeted cell, by avoiding points on sides that were close to neighboring cells preventing activation of neighboring cells. Preliminary experiments showed that stimulation spots had to be close to the soma to achieve reliable activation of the selected cell avoiding potentially confounding dendritic activation of cells. Thus, while our stimulation might activate cells that were not loaded with $\mathrm{Ca}^{2+}$ indicator, or were out of the focal plane (and thus not observed), the number of potentially activated neurons is likely very small $(<5)$. The power was adjusted to achieve reliable activation of the targeted cell on successive trials and ranged from 50 to $80 \mathrm{~mW}$ at the back aperture. We imaged responses full field at $750 \mathrm{~ms} /$ frame or for ROIs at 50-550 ms/frame and averaged over five trials. Fluorescence changes were analyzed off-line (MATLAB) by calculating 
A

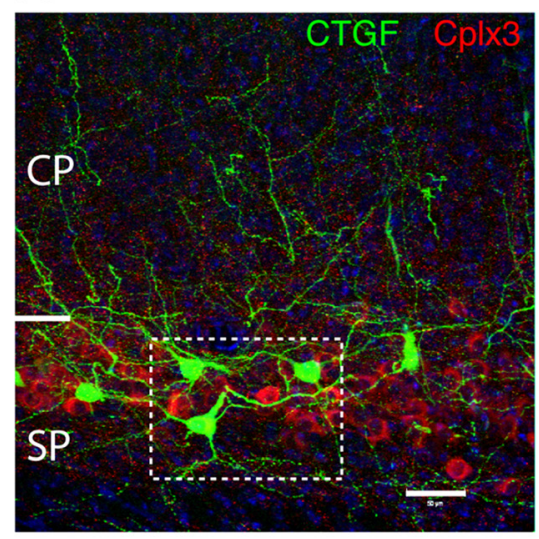

B

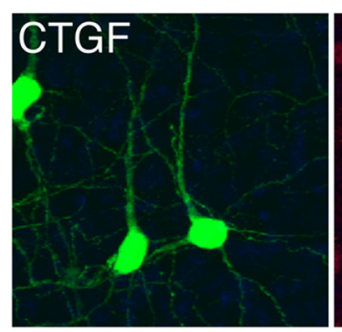

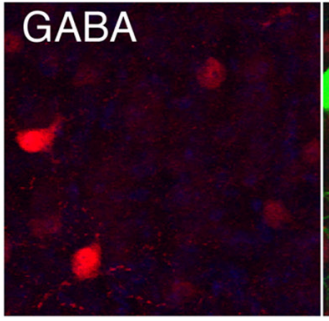


政



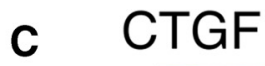


Figure 1. Subpopulations of SPNs have dendrites in the cortical plate. $A$, Colocalization of two markers of SPNs. Immunohistochemistry for complexin 3 (red) in CTGF-GFP animals (green). Blue stain is DAPI to highlight cell bodies. Note that CTGF cells are in the same lamina as Cplx3 cells but not all CTGF cells express complexin 3 (arrowheads indicate two CTGF + cells, one that did and one that did not costain with complexin3). Scale bar, $50 \mu \mathrm{m}$. The majority of GFP + cells ( $>80 \% ; n=98$ cells, $n=13$ fields) are located throughout the upper half of the subplate. $B$, CTGF cells are not GABAergic. Immunohistochemistry for GABA (red) in CTGF-GFP animals (green) shows that CTGF cells do not contain GABA. Scale bar, $50 \mu \mathrm{m}$. C, CTGF cells extend dendrites into the cortical plate. Shown are apical neurites from two (TGF-expressing SPNs (left and right pairs of panels). The neurites of both cells are studded with spines, suggesting that they are dendrites. The boxed region in the left panel of each pair is shown at higher magnification in the corresponding right panel. Scale bars (in each pair of panels): 5 and $1 \mu \mathrm{m}$, respectively.

relative change in fluorescence over an area encompassing the cell body (see Fig. 7A). A neuron was deemed responsive if significant ( $>95 \%$ confidence) $d F / F$ changes were detected for at least two subsequent frames and if its baseline fluorescence in the four frames before stimulation was stable. We performed these experiments at room temperature to reduce spontaneous activity and the chance of multisynaptic events.

Drugs. We use NBQX $(50 \mu \mathrm{M})$ to block AMPA currents, picrotoxin $(100 \mu \mathrm{M})$ to block GABA currents, and TTX $(1 \mu \mathrm{M})$ to block action potentials. All chemicals and drugs were obtained from Sigma-Aldrich.

Immunohistochemistry. Animals were deeply anesthetized with isoflurane and perfused transcardially with $4 \%$ PFA. The animal was decapitated and the brains were left in PFA for $1 \mathrm{~h}$ and rinsed three times with $1 \times \mathrm{PBS}$ and stored at $4^{\circ} \mathrm{C}$. Fifty-micrometer-thick sections were cut on a vibratome and blocked and permeabilized in a buffer containing $10 \%$ goat serum, 2\% BSA, and $0.3 \%$ Triton for $1 \mathrm{~h}$. All antibodies were diluted in this buffer. Sections were incubated in primary antibody [Cplx3 (Synaptic Systems; 1:3000); GABA (ImmunoStar; 1:1000)] overnight at $4^{\circ} \mathrm{C}$ and rinsed in $1 \times$ PBS with $0.1 \%$ Triton three times. Secondary antibody (goat anti-rabbit conjugated to AF594; Invitrogen) was added at a dilution of 1:500 and sections incubated at room temperature for $2 \mathrm{~h}$. Sections were rinsed as before and mounted in Vectashield under coverslip (Vector Laboratories).

Statistics. Results are plotted as means \pm SD as indicated. Populations are compared with ANOVA and Student's $t$ test. Differences are deemed significant if $p<0.05$.

\section{Results}

Molecularly identified SPNs extend dendrites into the cortical plate

SPNs are located in the future white matter below layer 6 and have diverse morphology (Kostovic and Rakic, 1980; Zhao et al., 2009; Kanold and Luhmann, 2010). We aimed to investigate whether SPNs receive inputs from the cortical plate. This can be achieved by either having SPN dendrites reaching into the cortical plate or by axons from superficial neurons projecting into the subplate. To investigate whether SPNs extend dendrites into the cortical plate, we studied a subset of SPNs that can be molecularly identified. SPNs show morphologic diversity, and recently a range of molecular markers for SPNs has been described (Hoerder-Suabedissen et al., 2009; Osheroff and Hatten, 2009). One marker is CTGF, which is selectively expressed in SPNs, especially in SPNs located more superficially (Heuer et al., 2003; Watakabe et al., 2007; HoerderSuabedissen et al., 2009). To ascertain whether CTGF-positive SPNs extend dendrites into the cortical plate, we used mice in which a sparse population of CTGF-positive SPNs were labeled with GFP [Tg(Ctgf-EGFP)156Gsat]. We first determined whether in these mice the GFP signal colocalizes with the other known subplate marker complexin3 (Hoerder-Suabedissen et al., 2009). Immunostaining for complexin 3 (Cplx3) in the CTGFGFP animals showed that GFP-positive cells were spatially comingled with complexin-positive cells and that some GFP-positive cells were also Cplx3-positive (Fig. 1A). However, the fraction of GFP-positive cells was much smaller than expected from the widespread CTGF expression (Heuer et al., 2003; Watakabe et al., 2007; Hoerder-Suabedissen et al., 2009; Osheroff and Hatten, 2009). Thus, the CTGF-GFP transgenic faithfully labels a subpopulation of SPNs. Interestingly, the CTGF-GFP-positive cells have diverse morphology (Fig. 1A); thus, CTGF likely defines a large class of SPNs. We next identified the transmitter phenotype of CTGF-positive neurons by immunostaining for GABA, which 
characterizes inhibitory neurons. We find that GFP-positive SPNs do not colabel with GABA ( 0 of 80 CTGF SPNs were GABA positive, and 0 of 119 GABA cells were CTGF positive; 12 fields; Fig. $1 B$ ). Thus, CTGF-positive SPNs are excitatory. We then investigated the targets and identity of neurites of GFP-positive neurons. We observed abundant CTGF-positive neurites in the cortical plate (Fig. $1 B, C$ ). Many of these neurites contained spines, suggesting that these were dendrites (Fig. 1C). Since spines are usually associated with excitatory synapses, these results suggest that CTGF-positive SPNs are likely to integrate synaptic inputs from all cortical layers by extending dendrites into the cortical plate.

\section{SPNs receive excitatory and inhibitory inputs from the cortical plate}

We next investigated functionally the sources of excitatory and inhibitory input to SPNs by whole-cell voltage-clamp recordings from SPNs $(n=75)$ in primary auditory cortex (A1) coupled with LSPS using caged glutamate (Shepherd et al., 2003). We recorded from cells between $\mathrm{P} 2$ and $\mathrm{P} 14$ to investigate eventual changes in SPN connectivity during key stages of auditory cortical development such as onset of hair cell function at P5, ear canal opening at P9, and the critical period ( $\sim$ P11-P13 in rat) (Shnerson and Pujol, 1983; Pujol et al., 1997; Ruebsamen and Lippe, 1997; de Villers-Sidani et al., 2007; Tritsch et al., 2010).

Photostimulation activates neurons, and if a connection is present between a stimulated neuron and the recorded neuron a PSC is visible in the recorded neuron. By varying the holding potential from -70 to $0 \mathrm{mV}$, the excitatory and inhibitory inputs to a given cell can be investigated. Figure $2 \mathrm{~A}$ illustrates this method for one SPN. During voltage-clamp recording when the SPN was held at $-70 \mathrm{mV}$, photostimulation can evoke two types of responses. The first type of response is characterized by a large inward current with short latency and is due to direct activation of the cell under study (direct response; Fig. $2 \mathrm{~B}$ ). The second type of response is characterized by a smaller amplitude inward current with longer latency (Fig. $2 B$ ). The longer latency currents could be abolished by addition of TTX (data not shown), indicating that presynaptic spiking activity was required. These responses were thus evoked EPSCs. When holding the SPN at a depolarized potential, direct responses are absent and only relatively long-lasting outward currents are observed (Fig. 2 B). These outward currents are evoked IPSCs and could be blocked by application of picrotoxin (data not shown). Some IPSCs had relatively short latencies, consistent with previous reports (Brill and Huguenard, 2009).

To identify the location at which neurons reside that project to a given SPN, we photostimulate at multiple locations (up to 900 locations) within a rectangular grid spanning the entire extent of the cortex from pia to ventricle to generate excitatory and inhibitory synaptic input maps. Figure $2 C$ shows traces from an example cell held at either -70 or $0 \mathrm{mV}$ to isolate excitatory and inhibitory inputs, respectively. This cell received both direct (gray traces) and synaptically evoked responses (black traces) from areas directly surrounding the cell body. The cell also received evoked responses from more superficial locations (yellow box). We measured the peak amplitude of the evoked response and superimposed the amplitude map onto the IR image of the slice to identify the spatial location of the inputs (Fig. 2D). The map of spatial location of inputs suggests that this SPN receives functional synaptic inputs upon stimulating superficial cortical layers including layer 4.

While LSPS is most effective in driving cells when the laser is targeted to somata, cells can potentially be activated when the laser is targeted to their dendrites, thus reducing spatial resolution. To test the functional resolution of LSPS at the studied ages, we obtained cell-attached recordings from layer 4 and layer 5/6 neurons from P4 to P14 ( $n=16$ and $n=19)$. We find that layer 4 neurons at all ages fired short latency $(<30 \mathrm{~ms})$ action potentials almost exclusively when the laser was targeted at layer 4 (Fig. $2 E$ ). In some layer 4 neurons, spikes were observed when layer $2 / 3$ or the subplate were stimulated, suggesting that both of these inputs can be functionally strong (arrow). We quantified these observations by calculating the distance from the soma at which action potentials could be elicited (Fig. $2 E$, right). These plots show that most action potentials were elicited if the stimulation spot was within $150 \mu \mathrm{m}$ from the soma (Fig. $2 E$, right, red dashed line). Layer 6 neurons are predominantly activated by stimulating within layer $5 / 6$ (Fig. $2 F$ ). While many layer $5 / 6$ neurons showed very restricted areas of activation (Fig. $2 F$, left) surrounding the cell body (Fig. $2 F$, right), others could also be driven by stimulating layer $2 / 3$ (Fig. $2 F$, right). This could be due to direct activation of the apical dendrite of the neuron or due to activation of a layer $2 / 3$ cell that has a strong synapse on the layer 6 neuron. Since the latencies of these distant evoked action potentials were very long $(37 \pm 10 \mathrm{~ms})$, the latter scenario is more likely. However, stimulation within layer 4 did not result in action potentials in most layer 5/6 neurons. To ascertain whether distant activation of layer 5/6 neurons could contribute to observed PSCs in SPNs after L4 stimulation, we plotted EPSCs and IPSCs evoked in SPNs by stimulating in layer 4. The EPSCs and IPSCs had a latency of $19.8 \pm 4.3$ and $20.3 \pm 4.3 \mathrm{~ms}$ (Fig. $2 G$ ). These latencies are comparable with the spiking latencies of layer 4 neurons when stimulated close to the soma (Fig. $2 E, G ; p>$ $0.05)$. However, these PSC latencies are much shorter than the latencies of layer 6 spikes evoked by stimulating away from the soma such as in layer 4 and $2 / 3$ (Fig. $2 F, G ; p<10^{-8}$ for distances $>200 \mu \mathrm{m}$ ). Thus, distant activation of layer $5 / 6$ cells at best only contributes a small fraction of observed layer 4 PSCs in SPNs. Thus, together, these data show that LSPS has laminar resolution and suggest that SPNs receive inputs from cortical neurons including from layer 4 neurons.

\section{Discerning two classes of SPNs based on the presence of cortical inputs}

Many SPNs received excitatory synaptic inputs from within the subplate and also significant inputs from cortical layers 1-4 (Fig. 3A-C). Cortical excitatory inputs to these SPNs originated from locations radial to the position of the recorded neuron, suggesting columnar organization (Fig. $3 B$ ). Inhibitory inputs to these cells originated within the subplate and also in superficial cortical layers (Fig. $3 B$ ). We quantified the laminar origin of intracortical excitatory and inhibitory inputs to SPNs by measuring the total synaptic current originating at each laminar (depth) position. This is done by integrating the responses for stimulus positions at the same depths. These profiles show a broad laminar region from which these SPNs received inputs (Fig. 3C). These profiles also show that inhibitory inputs were more restricted to locations closer to the recorded cell, evident as a more restricted profile for IPSCs. A second group of SPNs did not receive obvious inputs from cortical layers 1-4, but only from deep cortical layers and from within the subplate (Fig. $3 D-F)$; inhibitory inputs to these cells were similarly restricted to deep layers. These results suggest that two broad classes of SPNs exist as judged by inputs from layer 4 .

To validate our observation of functional synaptic inputs from superficial layers onto more superficial SPNs, we performed 

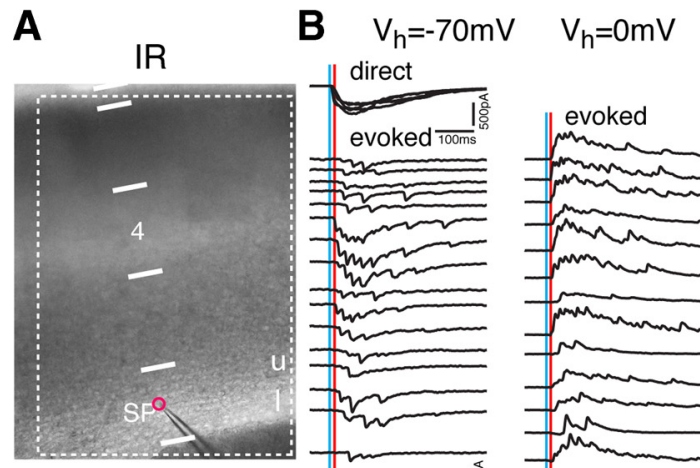

\section{C}
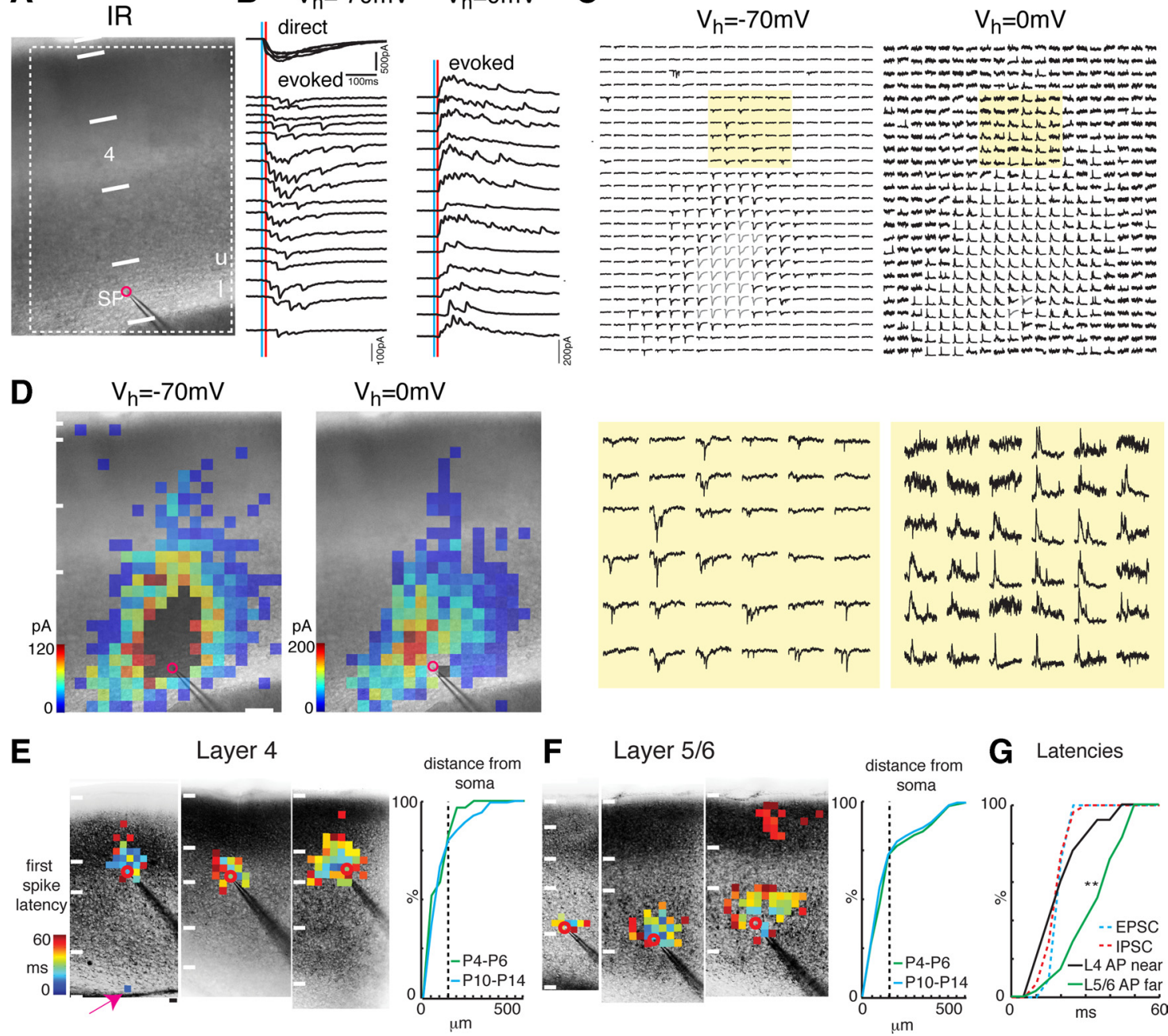

Figure 2. Laser-scanning photostimulation reveals cortical inputs to SPNs. $\boldsymbol{A}$, IR image of cortical field with patched SPN (pipette) that is being mapped. The box indicates the region of photostimulation. The borders between cortical layers based on IR image are indicated by white bars. The border between subplate and overlying layer 6 is characterized by a transition from horizontally oriented fibers and cells to a radial organization. The upper and lower subplates are indicated by "u" and "I." $\boldsymbol{B}$, LSPS performed at -70 and $0 \mathrm{mV}$ holding potential reveals excitatory and inhibitory evoked currents, respectively. Stimulation close to the cell body at $-70 \mathrm{mV}$ reveals short-latency large-amplitude direct responses. The blue lines indicate the time of the laser pulse. The red line indicates the longer latency of evoked responses versus the short latency of direct responses. C, Left, Traces obtained by LSPS in 500 stimulus locations for one cell held at $-70 \mathrm{mV}$. Traces showing a large-amplitude direct response are shown in gray. Note that large EPSCS can be observed at locations surrounding the direct response and at more superficial locations (highlighted in yellow box). Right, Traces obtained by LSPS in 500 stimulus locations for one cell held at $0 \mathrm{mV}$. Note that direct response is mostly absent. Note that large IPSCs can be observed at many locations. D, Maps of peak EPSC or IPSC transparently superimposed on the IR image of the slice. Colors indicate the amplitude. Locations resulting in a direct response are black. Note that EPCS and IPCS originate in locations surrounding the cell body, but also from more superficial locations including layers $2-4$. E, $F$, Cell-attached recordings from layer 4 and layer $5 / 6$ neurons. Plotted are maps of first spike latencies encoded in pseudocolor. Scale bar, $30 \mu \mathrm{m}$. Note that the shortest latencies are evoked close to the soma. Cumulative distribution on right show distance of stimulation locations where action potentials could be evoked for layer 4 ( $n=7$ at P4-P6 and $n=9$ at P10-P14) and layer $5 / 6$ cells ( $n=8$ at P4-P6 and $n=11$ at P10 -P14). Most effective locations were within 150 $\mu \mathrm{m}$ from soma in all cells (dashed black line). Note that, in layer $5 / 6$, neurons stimulation at locations $>400 \mu \mathrm{m}$ from the soma were able to cause action potentials. These were locations in layer $2 / 3$ and had long latencies (see cell on right in $\boldsymbol{F}$ ). Note the paucity of effective location in layer 4. G, Cumulative distributions showing the latency of EPSCs and IPSCs evoked by layer 4 stimulation in SPNs (dashed lines) and showing the latency of action potentials in L 4 neurons evoked by stimulation in L 4 close to the soma (black line) and action potentials in L5/6 neurons at P10 -P14 far from the soma [ $>200 \mu \mathrm{m}$, thus in layers $2 / 3-4(\mathrm{see} \boldsymbol{F})$; green line]. Latencies for evoking action potentials in layer $5 / 6$ cells when stimulating at distal locations were significantly longer than latencies for PSCs in subplate with layer 4 stimulation $\left({ }^{* *} p<10^{-8}\right)$.

LSPS recordings in CTGF-GFP animals. CTGF is a marker of the superficial subplate neurons (Heuer et al., 2003; HoerderSuabedissen et al., 2009; Osheroff and Hatten, 2009) (Fig. 1). Recording from cells that were located in close proximity to GFPexpressing cells ( $n=5$ cells; Fig. $3 G)$ showed excitatory and inhibitory inputs from the overlying cortex (Fig. $3 H$ ).

To investigate the origin of the multiple classes of input patterns to SPNs, we quantified the amount of EPSC and IPSC originating in layer 4 . We determined the position of layer 4 by three methods. First, the position of layer 4 was identified from the DIC images (Fig. $4 A$ ). Figure $4 A$ shows the relative location of layer 4 across the different ages. As a second method, we used the expression of laminar-specific markers (Hooks et al., 2011). We used the expression profile of a specific marker of layer 4 in sections that closely resembled our slice plane (ROR- $\alpha$; images from the Allan Brain Atlas 100054927; NCBI: NM_013646) (Fig. 4A, right). The estimated layer boundaries at young (P2-P6) and older (P10-P14) ages are within the range of ROR- $\alpha$ expression at $\mathrm{P} 4$ and P14 (Fig. $4 \mathrm{~A}$, right). In addition, we used transgenic mouse line expressing cre recombinase under the control of Scnnla promoter. Upon crossing with another transgenic line expressing td tomato in a cre-dependent manner, the resultant 


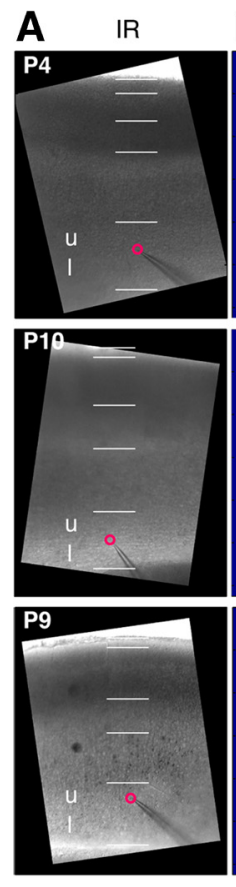

G

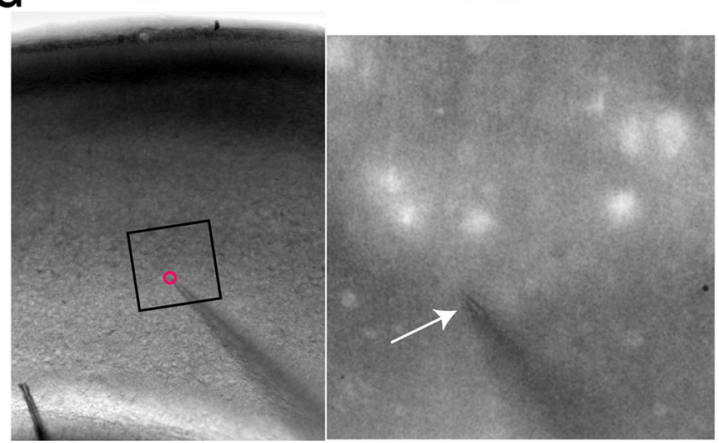

B $V_{h}=-70 \mathrm{mV}$
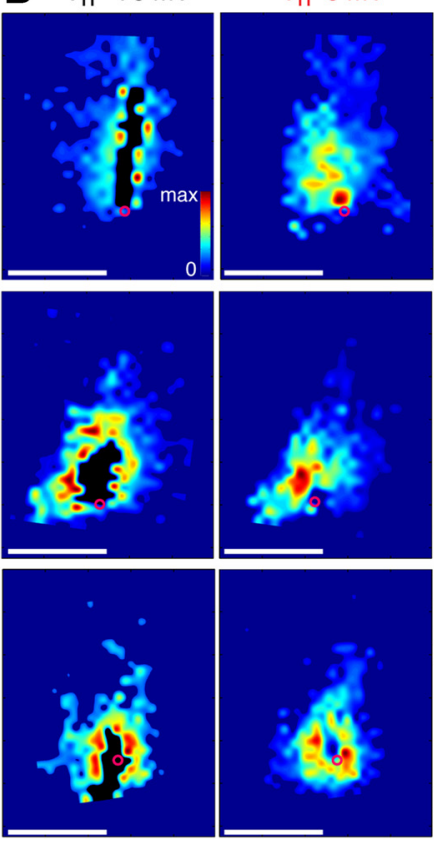

CTGF
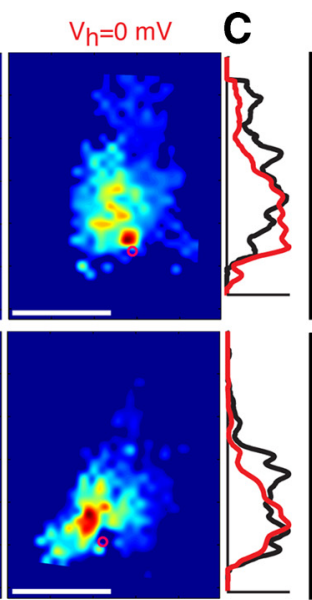
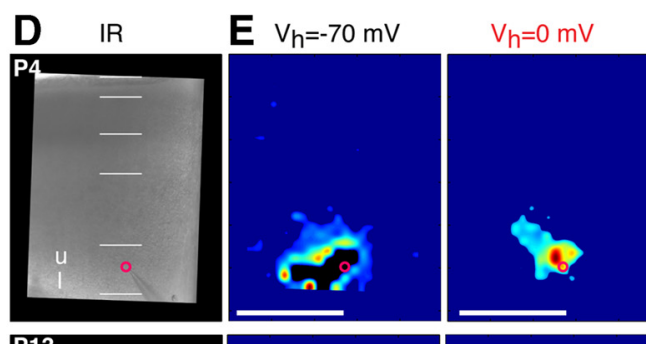

$\mathbf{F}$
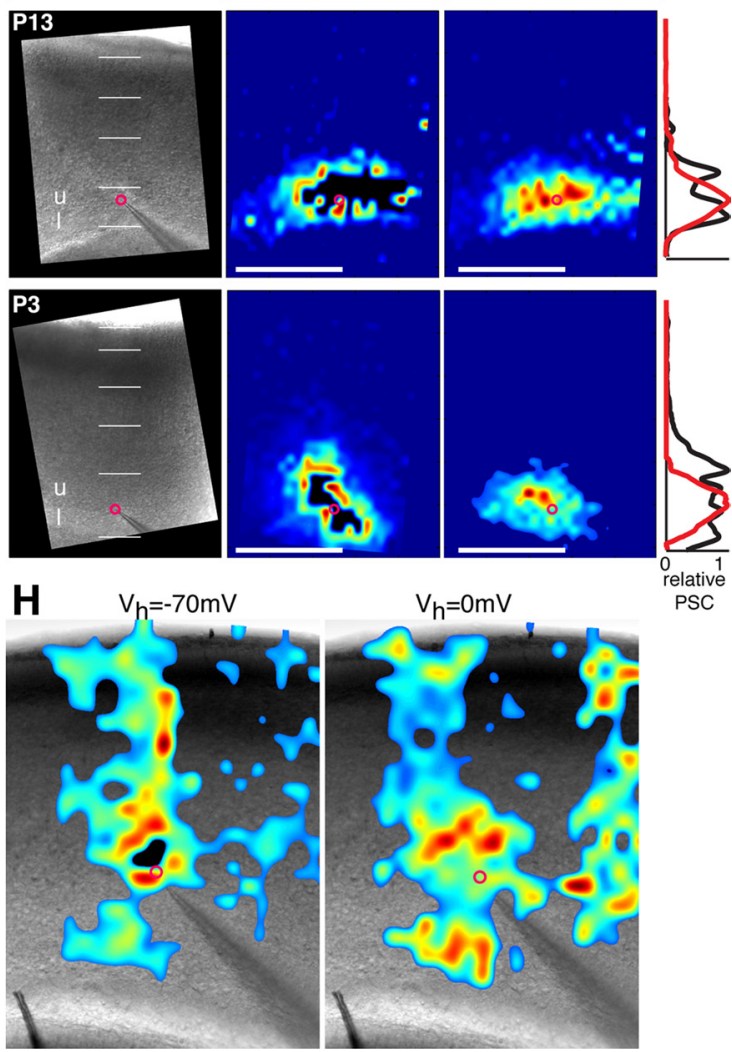

Figure 3. A population of SPNs receives excitatory and inhibitory inputs from layers $2-4$. A-C, Three examples of SPNs that show EPSCs and IPSCs evoked by stimulation of layer 2-4.A, Biocytin and IR pictures of recording location in the subplate. $\boldsymbol{B}$, EPSC maps generated by holding the neuron at $-70 \mathrm{mV}$ and IPSC maps generated by holding the neuron at $0 \mathrm{mV}$. Maps are overlaid on the IR image of the cortex. Scale bar, $500 \mu \mathrm{m}$. C, Traces show summed EPSC (black) and IPSC (red), respectively, along the cortical (laminar) depth. Traces are normalized to the maximum and are aligned with the corresponding maps and images in $\boldsymbol{B}$. Note that a large fraction of inputs originates in superficial layers. $\boldsymbol{D}, \boldsymbol{E}$, Three examples of SPNs that do not show EPSCs and IPSCs evoked by stimulation of layer 2-4. D, Biocytin and IR pictures of recording location in the subplate. E, EPSC maps generated by holding the neuron at $-70 \mathrm{mV}$ and IPSC maps generated by holding the neuron at $0 \mathrm{mV}$. Maps are overlaid on the IR image of the cortex. $F$, Traces show summed EPSC (black) and IPSC (red), respectively, along the cortical (laminar) depth. Traces are normalized to the maximum and are aligned with the corresponding maps and images in $\boldsymbol{E}$. Note the absence of inputs originating in superficial layers. $\boldsymbol{G}, \boldsymbol{H}$, LSPS in CTGF-GFP mice to identify subplate location. $\boldsymbol{G}$, DIC image of slice; region inside the box is shown in the higher-magnification fluorescence image to the right. Note that fluorescent cells are located more superficially than the recording electrode (arrowhead indicates electrode tip). Thus, the recorded cell was a SPN. H, Smoothed LSPS maps (see methods) of this cell, showing excitatory and inhibitory inputs from superficial cortical layers.

progeny exhibit dense layer 4 label. We then cut thalamocortical sections and examined the extent of layer 4 by measuring the RFP signal profile. We find that RFP signal was present in areas closely matching our estimated layer boundaries from DIC (Fig. 4B). Thus, our estimate of layer 4 position from DIC is within the range of expression of layer 4-specific markers.

We next quantified the strength of inputs from layer 4 . While peak amplitude indicates synaptic strength, stimulation at a particular location can evoke events of different durations. Thus, a second measure of the strength of the functional connectivity is the integrated charge of the PSC. We quantified both the peak amplitude and integrated charge of the detected events. We then calculated both the total and fractional charge and amplitude, respectively, by dividing the amplitude or charge originating in layer 4 by the total amplitude or charge that each cell received from all locations. Both the total integrated charge and peak amplitude for the EPSC and IPSCs originating in layer 4 were corre- lated with the fractional charge and amplitude (Fig. 4C,D). In addition, fractional peak and charge were well correlated for the EPSC and IPSC indicating that both measures could be used to compare the inputs originating in the different layers. The lower correlation values for IPSCs might be due to larger variability in the time constants of GABAergic currents.

The distribution of both the fractional excitatory charge and amplitude that originate in layer 4 for each neuron showed a prominent peak at zero and a population of cells at nonzero values (Fig. 5A). This suggested that the distribution contained two populations. To explore the existence of multiple populations, we performed $k$-means clustering. Clustering showed that $>70 \%$ of the variance in the data could be explained by two clusters, while further clusters only slightly increased the explained variance (Fig. 5B), suggesting the existence of two populations of SPNs. Thus, while many SPNs receive little layer 4 input, others receive large layer 4 inputs (vertical dashed line 


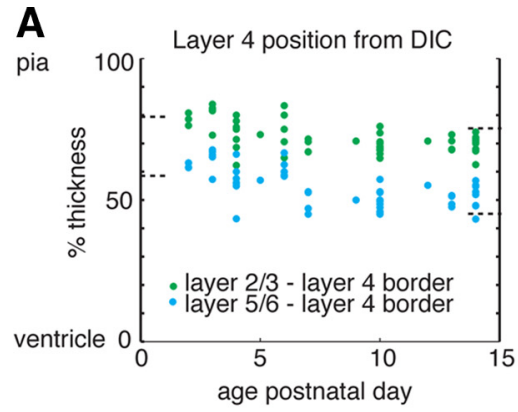

C
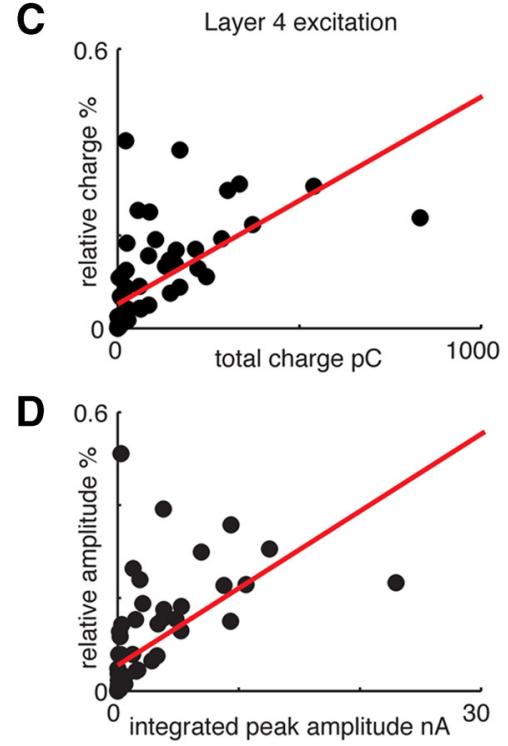

E

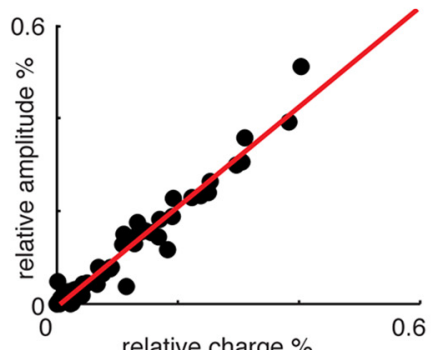

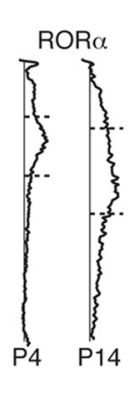

B
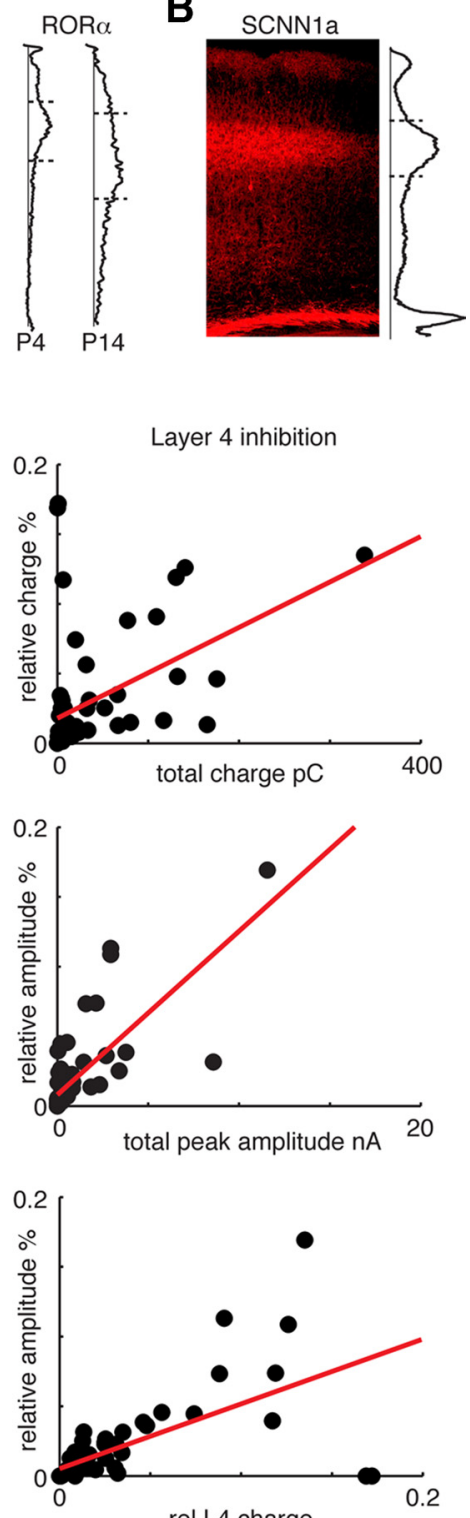

rel L4 charge
0.2

Figure 4. Cortical input from layer 4 to SPNs. A, Upper and lower borders of layer 4 for each mapped SPN determined from IR pictures. The locations are relative to the distance between ventricle (0\%) and pia (100\%). Note that the relative location of layer 4 shifts downward over development as layer $2 / 3$ matures. The traces on the right indicate expression profiles of the layer 4 marker ROR- $\alpha$ (images from Allen Brain Atlas image series ID 100054927; images 101080073-7 and 100825083-7) in sagittal slices from mice at P4 and P14. Note that the peaks of the expression profiles approximately match the locations of layer 4 determined from DIC. B, Layer 4-specific RFP signal in thalamocortical slices of A1 at P11. RFP expression obtained by crossing the layer 4 driver line SCCN1a-Cre with flox-RFP. Expression profile on right shows peak that matches the locations of layer 4 determined from DIC. C, Total and relative integrated charge for EPSCS and IPSCS evoked by layer 4 stimulation. Total charge is calculated by summing the charges of each PSC at each stimulation site that gave rise to a PSC in layer 4. Relative charge is calculated by dividing by the total charge the cell receives from all layers. The red lines indicate linear regression $\left(r^{2}=0.39, r^{2}=0.2\right.$ for left and right panels, respectively). $\boldsymbol{D}$, Total and relative peak amplitude for EPSCs and IPSCs evoked by layer 4 stimulation. Total amplitude is calculated by summing the peak amplitudes of each PSC at each stimulation site that gave rise to a PSC in layer 4. Relative amplitude is calculated by dividing by the total amplitude the cell receives from all layers. The red lines indicate linear regression $\left(r^{2}=0.33, r^{2}\right.$ $=0.54$ for left and right panels, respectively). $\boldsymbol{E}$, Relative integrated charge and amplitude for EPSCs and IPSCs evoked by layer 4 stimulation. The red lines indicate linear regression $\left(r^{2}=0.96, r^{2}=0.4\right.$ for left and right panels, respectively).

indicates the border between the two clusters). Similarly, the distribution of fractional inhibitory charge or amplitude also showed the existence of two populations of SPNs. So far, we considered groups of SPNs either by their EPSCs or by their IPSCs. To investigate whether these groups of SPNs represented individual neurons that received both excitatory and inhibitory input from layer 4 , we performed $k$-means clustering on both layer 4 fractional excitatory and inhibitory charges/amplitudes (Fig. $5 B$, bottom) and found the presence of two groups. Together, these results suggest that there are two populations of SPNs: one that receives input from layer 4 and one that does not.

\section{Changing patterns of laminar inputs to SPNs over development}

We previously found that the thalamic input to SPN changes over development in that SPNs receive increasing input from the thalamus with age (Zhao et al., 2009). We therefore investigated whether the different spatial input patterns from layer 4 reflected developmental changes. SPNs were grouped over three age ranges ( $\mathrm{P} 2-$ $\mathrm{P} 5, \mathrm{P} 6-\mathrm{P} 9$, and $\mathrm{P} 10-\mathrm{P} 14)$, approximately corresponding to distinct functional states of the auditory system. Hair cells do not become functional before P5, and thus only spontaneous evoked activity is present in the auditory system (Tritsch et al., 2010). After onset of hair cell function but before the opening of the ear canal, spontaneous and high-threshold soundevoked responses might occur. In mice of the C57BL/6 strain, the ear canal opens at P9 (Shnerson and Pujol, 1983; Pujol et al., 1997; Ruebsamen and Lippe, 1997). After ear opening, low-threshold hearing develops, followed by an early critical period in which experience can alter the tuning of cells ( P11-P13 in rat) (de Villers-Sidani et al., 2007).

Plotting the distribution of fractional excitatory or inhibitory charge showed that the numbers of cells receiving excitatory layer 4 input increased over development (Fig. 5C). Plotting the fraction of SPNs that did or did not receive large excitatory input over the three developmental stages showed that the fraction of cells receiving large excitatory layer 4 inputs increased over development (Fig. 5D). While for excitatory inputs using integrated charge and peak amplitude did not result in any differences, for inhibitory inputs there are differences. The size of the fraction of SPNs that received inhibitory inputs from layer 4 possibly increased when using peak amplitude but stayed constant when using charge (Fig. 5D). This difference might be due to maturational changes in intracortical inhibition. We next investigated whether the emergence of excitatory and inhibitory inputs are linked by clustering on both layer 4 fractional excitatory and inhibitory inputs (Fig. 5C). Again, clustering shows the existence of two distinct populations of SPNs with the population that receives layer 4 input increasing in number (Fig. $5 D$ ), suggesting that excitatory inputs dominate the cluster- 

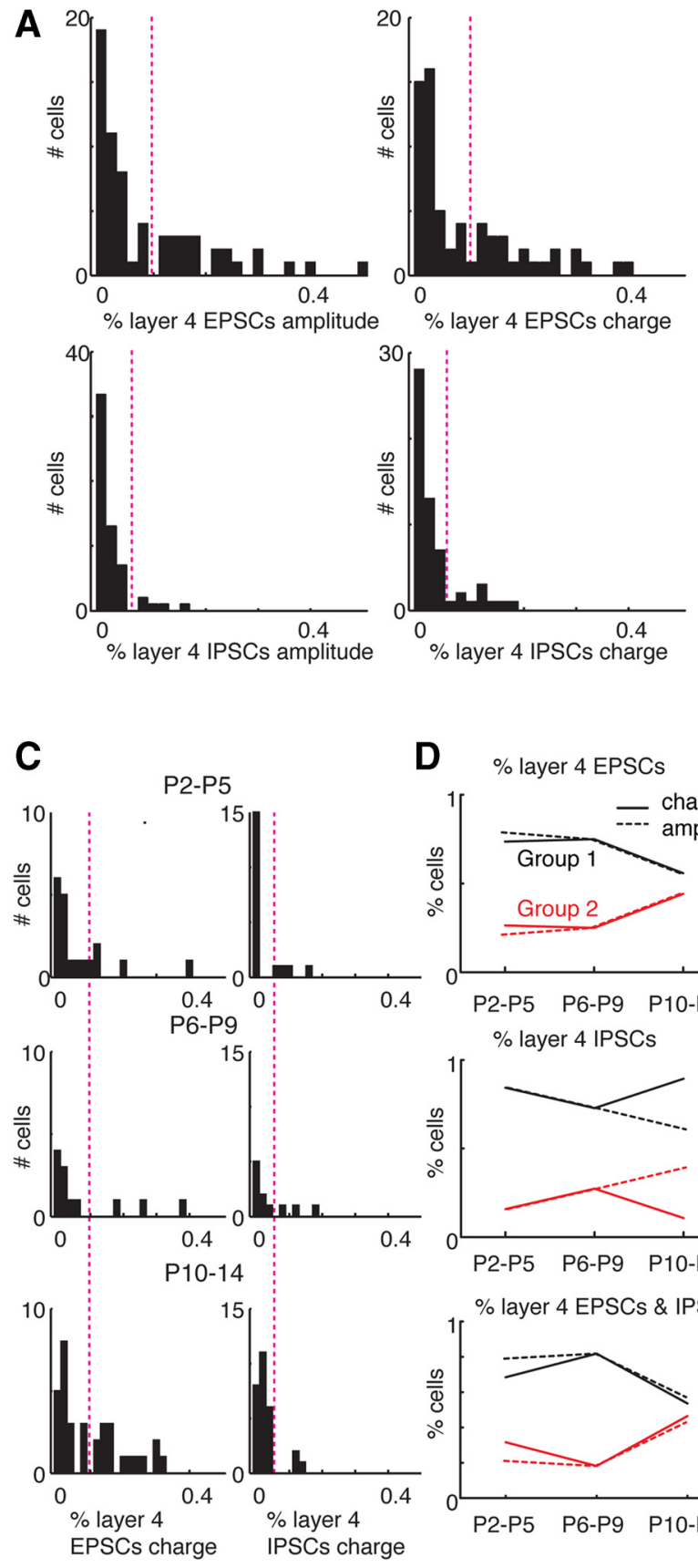

B
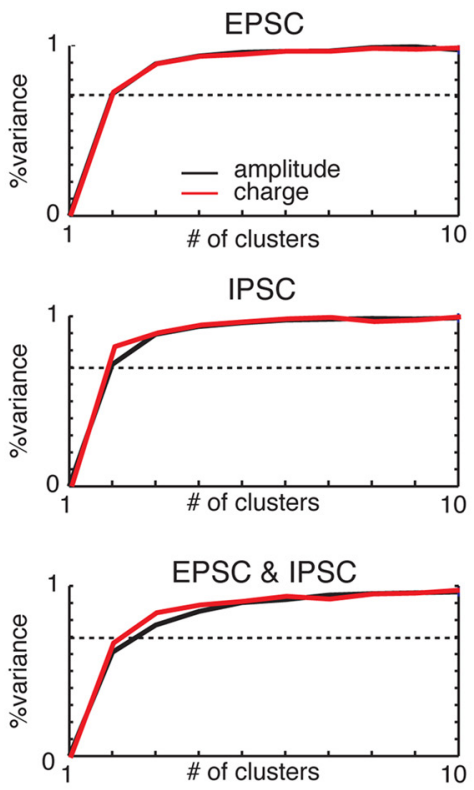

$\mathbf{E}$
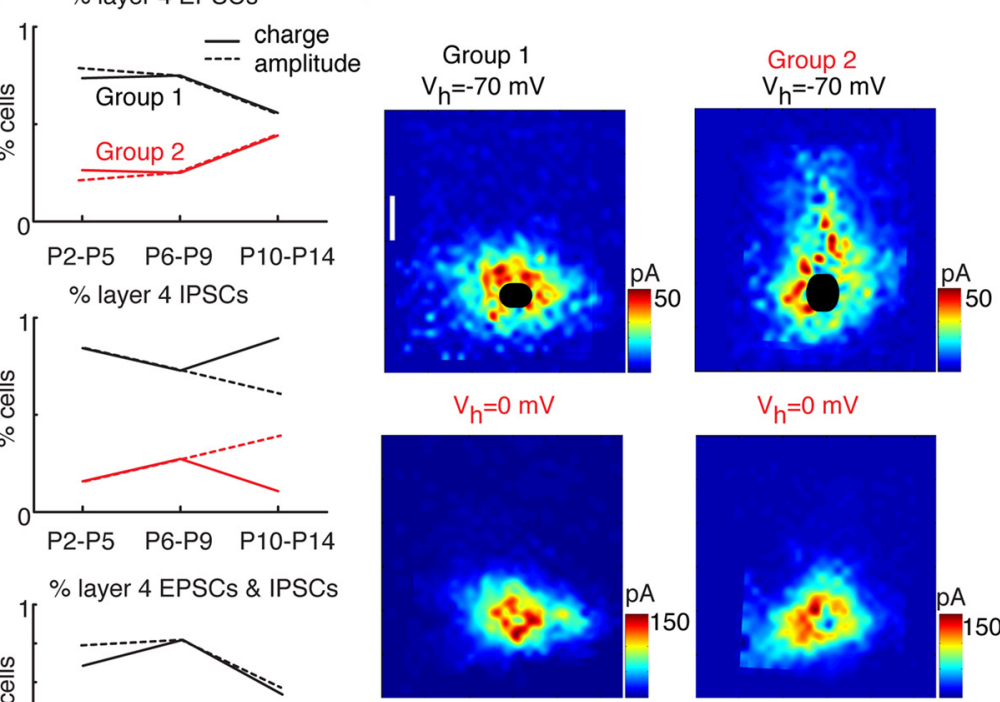

Figure 5. Cortical input to SPNs develops in the second postnatal week. A, Histograms of relative amplitude (left) and charge of EPSCs (top) and IPSCs (bottom) originating from layer 4 stimulation in individual SPNs. The vertical dashed lines indicate intersection between the two distributions obtained with $k$-means clustering. $\boldsymbol{B}$, Fraction of total variance explained by $k$-means clustering with different numbers of clusters. Graphs show clustering on relative EPSC charge and amplitude (top), relative IPSC charge and amplitude (middle), or on both EPSC and IPSC charge and amplitude (bottom). The horizontal dashed line indicates 70\%.C, Histograms of integrated charge of layer 4 EPSCs and IPSCs in the different age groups. Note that, at young ages, only few cells had large inputs from layer 4. The vertical dashed line indicates where the whole population separates (see A). D, Age distribution of SPNs without (black; Group 1) or with (red; Group 2) layer 4 EPSC (top), IPSC (middle), or EPSC and IPSC (bottom) input. The solid lines show age distribution based on integrated charge, while the dashed lines show distribution based on peak amplitude. E, Left, Average LSPS map for SPNs within Group 1 at older ages (P10 -P14). Cells were aligned to their soma position. Black indicates direct responses. Note that most inputs originate from close to the cell body and that the amplitudes of IPSCs are larger than those of EPSCS. Scale bar, $200 \mu \mathrm{m}$. Right, Average LSPS map for SPNs within Group 2 at older ages (P10 -P14). Cells were aligned to their soma position. Black indicates direct responses. Note that large excitatory inputs originate from locations distal from the cell body in layer 4.

ing. Together, our data suggest that while most SPNs at young ages do not receive inputs from superficial layers, a substantial fraction at older ages do receive excitatory and inhibitory inputs from superficial cortical layers, including layer 4 . Figure $5 E$ shows the input maps for the two groups present at older ages.

To validate the existence of cortical inputs to SPNs, we used a second independent measure. We calculated the laminar extent of inputs from the cortical plate to each SPN by measuring the
$20 \%$ peak width of the laminar spatial distribution of PSCs (Fig. $6 \mathrm{~A}$ ) and subtracted the distance of the soma from the layer 6 border. This results in a measure of the laminar extent of inputs only within the cortical plate. A small extent indicates inputs from only deep layers, while a large extent indicates input from deep and superficial layers. As expected, the excitatory laminar extent correlates with the amount of inputs from layer 4 (Fig. $6 B$ ). In other words, cells that had layer 4 input (Group 2) also 

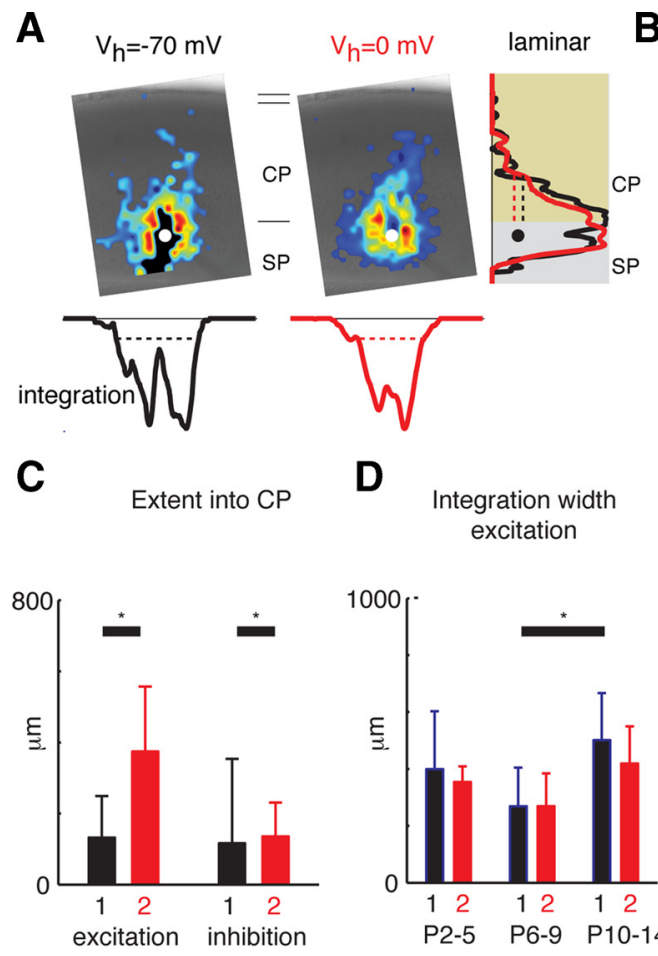

D

Integration width excitation

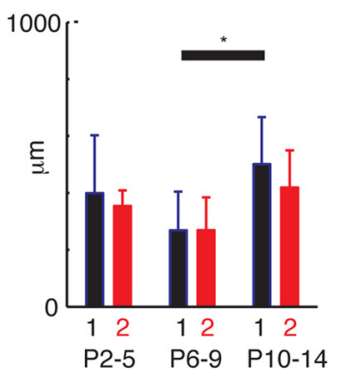



E

Integration width inhibition

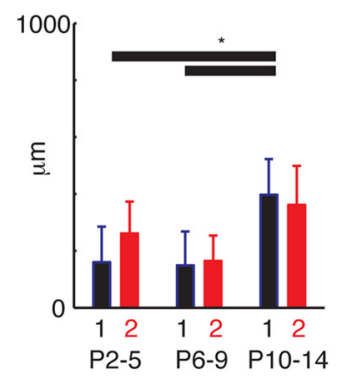

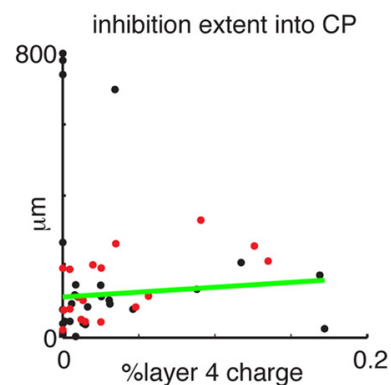

F

mean layer 4 amplitudes excitation inhibition

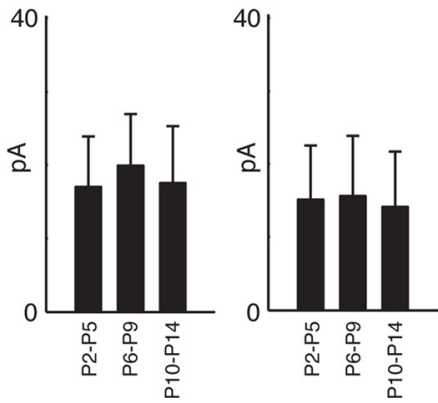

Figure 6. Columnar and laminar extent of inputs increases with age. A, EPSC and IPSC maps overlaid on the IR image of the cortex. Traces on the right side and bottom show summed EPSC (black) and IPSC (red) along the cortical (laminar) depth (right) and along the columnar extent (bottom). Traces are normalized to the respective maxima and aligned with the maps. Note the "dips" in the traces from the excitatory map indicating areas that showed direct responses (close to cell body, white dot), where no EPSC could be measured. Laminar or columnar widths are derived from measuring the $20 \%$ widths (dashed lines). $\boldsymbol{B}$, Extent of excitation in the cortical plate (extent $=$ laminar width - distance of cell body from layer 6 border; dashed lines in $\boldsymbol{A}$ ) is correlated with the relative PSC amplitude from layer 4. Plotted are relative EPSC (left) and IPSC (right) amplitude from layer 4 versus excitatory (left) or inhibitory (right) laminar width for all SPNs (Group 1 indicated in black; Group 2 in red). The green lines indicate regression fits of the data. Note the significant correlation of relative layer 4 EPSC charge and excitatory laminar width $\left(r^{2}=0.49\right)$, and the weaker correlation of relative layer 4 IPSC charge and inhibitory laminar width $\left(r^{2}=0.004\right)$. C, The average excitatory of Group 2SPNs (red; with layer 4 inputs) is larger than the extent for Group 1 SPNs (black; that did not show layer 4 inputs) $(p<0.001)$, while the inhibitory extent is only slightly larger $(p=0.048)$. Plotted are means $+S D$. D, Columnar width of excitation for Group 1 (black) and Group 2 (red) SPNs. Plotted are means + SD. There was a slight increase between P6 and P14 for Group $1(p<0.05)$. E, Columnar width of inhibition for Group 1 and Group 2 SPNs. Plotted are means + SD. The columnar width of inhibition of Group 1 SPNs increases between P2 and P14 as well as between P6 and P14 $(p<0.05)$. $\boldsymbol{F}$, Average amplitude for EPSCs and IPSCs originating in layer 4 at different ages. Plotted are means + SEM. Amplitudes do not increase over development ( $p>0.1 ;$ ANOVA).

showed more inputs from a larger extent (up to $700 \mu \mathrm{m}$ above the layer 6/subplate border) of the cortical plate. Plotting the average cortical input extent of all SPNs (Fig. 6C) showed that the cells receiving layer 4 inputs also showed a laminar extent of $>500$ $\mu \mathrm{m}$, which encompasses most of the thickness of mouse cortex and most certainly encompasses layer 4 (Hooks et al., 2011). This analysis also showed that the correlation and laminar extent for inhibitory inputs was smaller than that of excitatory inputs (Fig. $6 B, C)$ - consistent with a smaller fraction of inhibitory inputs from layer 4 (Fig. 5). Together, these analyses confirm that a subset of SPNs receives both excitatory and inhibitory input from the superficial cortical plate.

SPNs integrate cortical inputs in a laminar area from extending their cell body through the laminar extent of cortex, thus from locations putatively within the same column. In addition, the LSPS maps show that SPNs also integrate information from other SPNs and layer 6 across a large cortical area perpendicular to the laminar axis. Thus, SPNs potentially integrate information across cortical columns. To identify whether the two groups of SPNs showed any systematic differences in their columnar spatial integration, we thus quantified the spatial organization of cortical inputs to individual SPNs perpendicular to the radial axis. The SPN inputs in this axis are dominated by intra-subplate inputs (Figs. 2, 3). We measured the extent of inputs from cortical locations to SPNs for each cortical layer by measuring the EPSC and
IPSC amplitude parallel to the pial surface (Fig. $6 \mathrm{~A}$ ). We find that both populations of SPNs integrate inputs from 200-1000 $\mu \mathrm{m}$ (Fig. 6D). The extent of this integration for EPSCs and IPSCs in Group 1 SPNs increased over development (Fig. 6D,E). These results suggest that SPNs undergo developmental expansion of their inputs.

The observed developmental increase in total EPSC amplitude (or charge) and in relative EPSC amplitude (or charge) from layer 4 could be due to increases in EPSC amplitude evoked at any location or from an increase in the number of locations that evoked EPSCs. To distinguish between these possibilities, we plotted the mean EPSC and IPSC amplitudes resulting from each stimulation location in layer 4. The amplitude of mean EPSCs and mean IPSC evoked by stimulation in different locations at different ages was similar ( $p>0.1$; Fig. $6 F$ ). Together, these results suggest that increases in layer 4 input are due to an increased number of synaptic connections from layer 4 .

These results suggest that there exist at least two different classes of SPNs that integrate cortical information differently. One class receives predominantly deep input from layer $5 / 6$ and the subplate, and the other population receives deep and superficial input. In particular, this latter class of SPNs receives inputs from cells in layer 4, which is one of the target layers of SPN projections. Thus, over development, a feedback circuit between layer 4 and subplate is established. In addition, these data suggest 


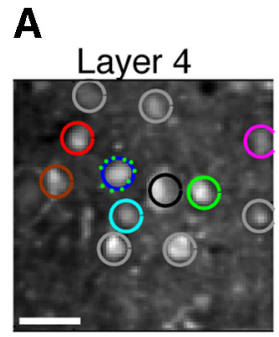

D

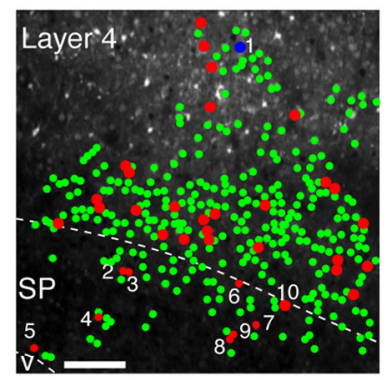

B Distance

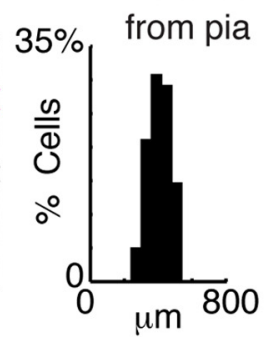

C
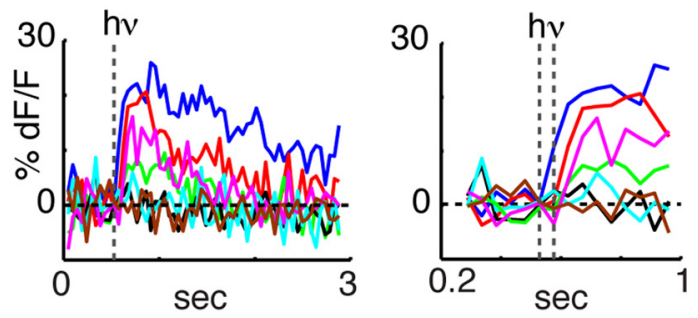

E

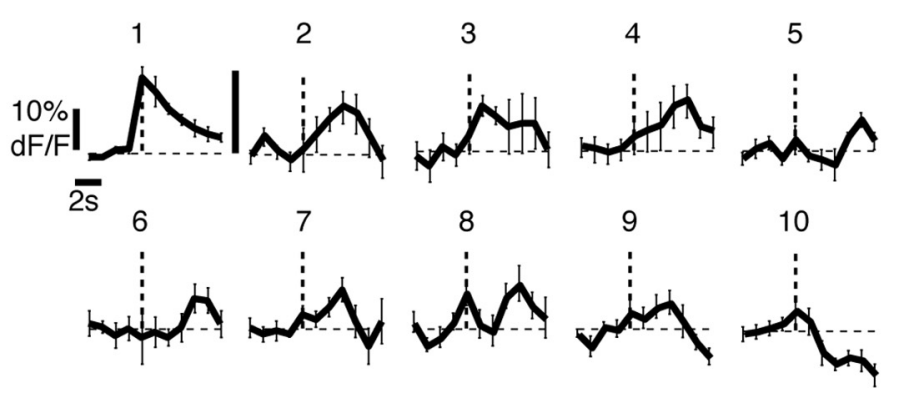

Figure 7. Layer 4 neurons influence a subpopulation of cells in the subplate. $A$, Two-photon photostimulation combined with two-photon $\mathrm{Ca}^{2+}$ imaging. Shown is an image of layer 4 neurons loaded with OGB-1 AM. Scale bar, $20 \mu \mathrm{m}$. The circles indicate the area over which fluorescence was measured. The green dots indicate stimulation locations around the blue cell. $\boldsymbol{B}$, Histogram shows location of stimulated neuron relative to pia. Stimulated neurons were located at $407 \pm 70 \mu \mathrm{m}$ from the pia, and thus are located in the middle of layer 4 . $\boldsymbol{C}$, Graphs show fluorescence changes in seven cells in layer 4 after stimulation of blue cell (stimulation time indicated by " $\mathrm{h} \nu$ " and vertical dashed line). Frame duration was $48.8 \mathrm{~ms}$. Note large fluorescence increases in stimulated cell (blue) and smaller shorter duration increases in three other cells. Note that many cells in close vicinity to the stimulated cell did not show any $\mathrm{Ca}^{2+}$ increases. The right graph shows the responses at the onset of stimulation. $\boldsymbol{D}$, Stimulation of layer 4 neuron results in fluorescence changes in cells in cortical plate and subplate. Fluorescent image and overlay showing location of stimulated neuron (marked blue) and imaged cell bodies in cortical plate and subplate. Imaged but not responding cells are marked green, while responding cells are marked red. Scale bar, $100 \mu \mathrm{m}$. The white dashed line indicates layer 6/subplate boundary estimated from DIC. Ventricle is indicated by "v." $E$, Traces show fluorescence transients (mean \pm SEM) in stimulated cell (" 1 ") and responding cells in subplate ("2" to " 10 "). Calibration bar indicates $10 \%$ dF/F and applies to all traces. The dashed vertical line indicates time of layer 4 stimulation. While many cells showed fluorescence increases, cells could also show fluorescence decreases or combinations of increases and decreases (i.e., cells 9 and 10). This could be due to the potential activation of more than one layer 4 neuron, different receptors in postsynaptic cells, activation of inhibitory neurons, or intrinsic differences between cells.

that there is substantial remodeling present in subsets of SPNs during the postnatal period.

Layer 4 neurons influence a subset of cells in the subplate Our data show that layer 4 neurons signal to SPNs. How many SPNs does a layer 4 neuron signal to? While LSPS with singlephoton excitation has laminar resolution (Fig. 2), it is likely that multiple neurons are stimulated at each stimulus location. Thus, we cannot estimate the connectivity probability of the layer 4 projections to SPNs. Thus, to estimate how many SPNs a layer 4 neuron signals to, we need to increase spatial resolution. The spatial selectivity can be increased by limiting the volume and location of stimulation by using two-photon photostimulation (Nikolenko et al., 2007). By choosing stimulation spots surrounding cell bodies, only one or very few cells can be stimulated. To measure the responses in a population of SPNs, we used twophoton $\mathrm{Ca}^{2+}$ imaging by loading slices with OGB-1 AM (Fig. $7 A$ ). While imaging sacrifices temporal resolution, it allows us to monitor a large number of SPNs. Stimulating layer 4 cells (Fig. $7 B)$ by two-photon photostimulation $(n=62$ targeted cells in slices from 7 animals; P7-P14) reliably leads to large $\mathrm{Ca}^{2+}$ increases in the targeted cell and slightly delayed $\mathrm{Ca}^{2+}$ increases in a subpopulation of nearby cells while other cells in the vicinity did not show fluorescence changes (Fig. 7C). This suggests that our stimulation reliably activates the targeted cells and confirms that layer 4 cells are connected to other layer 4 cells. We then imaged a larger area (up to $594 \times 594 \mu \mathrm{m}$ ) that encompasses layer 4 and subplate while stimulating layer 4 neurons. We find that, after stimulation of layer 4 neurons, a number of imaged neurons showed fluorescence changes (Fig. 7D, labeled red). Responding cells included cells in the subplate $(400-600 \mu \mathrm{m}$ deeper than layer 4) (traces for responding cells in subplate shown in Fig. 7E). Of 5440 imaged subplate cells, 407 (7.5\%) showed fluorescence changes following layer 4 stimulation. The time courses of the fluorescence changes in subplate cells were diverse (Fig. 7E), possibly reflecting differences in connection strength, receptor composition, and intrinsic characteristics of the cells. The obtained values of connection probabilities are likely an underestimate because two-photon imaging can only monitor neurons within a thin optical $z$-section. In addition, $\mathrm{Ca}^{2+}$-imaging more reliably reports strong connections that evoke large fluorescence changes, while weak inputs will not be observed. Responding cells could be found at lateral distances of up to $\pm 200 \mu \mathrm{m}$ (Fig. $7 D$ ) from the stimulated cell. However, because our imaging area is limited to $\sim 600 \times 600 \mu \mathrm{m}$, we might have missed potential responding cells further away. Nonetheless, together with the LSPS data, these results suggest the existence of a connection between layer 4 and SPNs that can extend at least $200 \mu \mathrm{m}$ along the columnar axis.

\section{Laminar organization of SPNs respective to input patterns}

Our data show that some SPNs receive inputs from the cortical plate, while others do not. What then is the difference between these cells? Histologically, the subplate zone is not uniform. The subplate encompasses cells in an upper, relatively more cell dense, sublamina below layer 6 (future layer $6 \mathrm{~b}$ ), as well as 


\section{location within subplate}

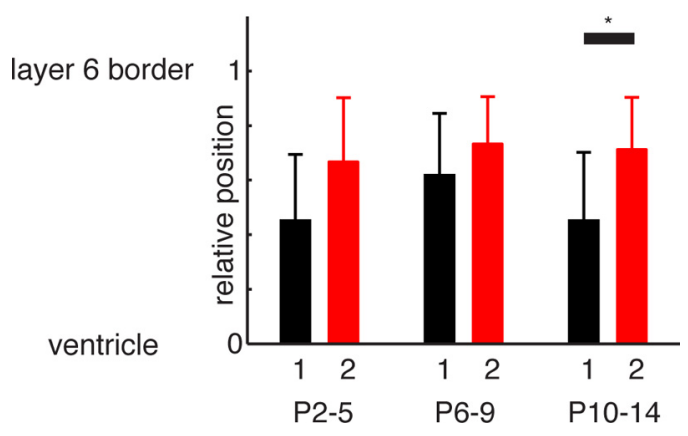

Figure 8. Laminar differences within subplate. The bar graph shows the location (mean + SD) of Group 1 and Group 2SPNs within subplate at different ages. The location of the subplatelayer 6 border is indicated by 1 , and the ventricle is at location 0. At P10 -P14, Group 2SPNs that receive layer 4 inputs are located closer to the pia than Group 1SPNs that do not $(p<0.05)$.

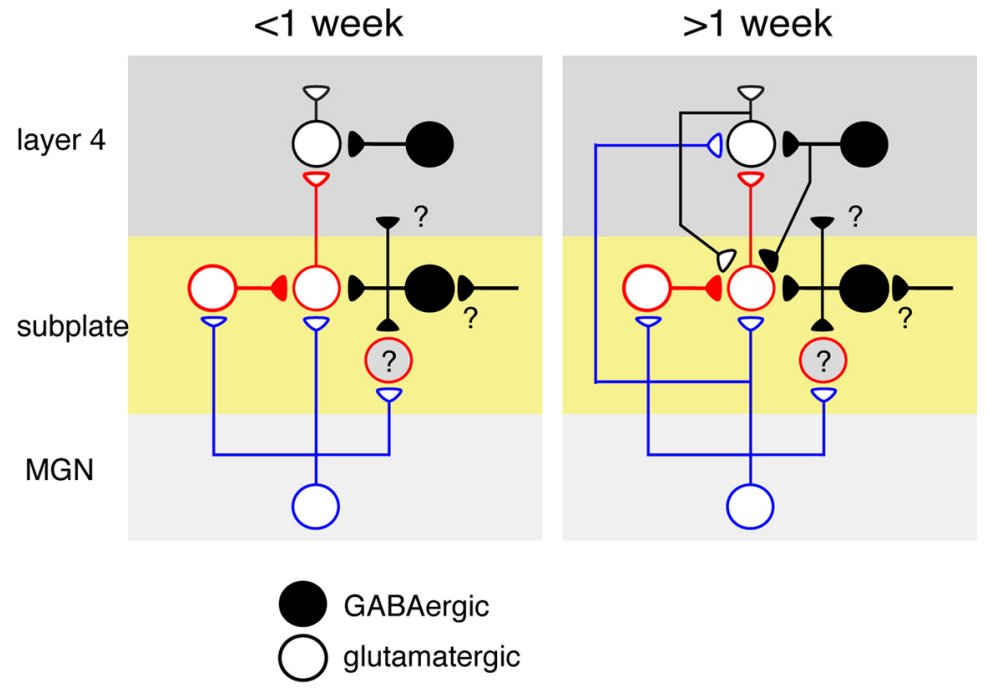

Figure 9. Model circuit of SPNs in auditory cortex. Excitatory SPNs receive input from MGN, while inhibitory SPNs do not receive inputs from MGN but from other unknown sources ("?"). Excitatory SPNs project to layer 4 and to other SPNs, while the projection targets of inhibitory SPNs are unknown. In the second postnatal week, superficial SPNs start to receive excitatory and inhibitory feedback connections from layer 4, while deep SPNs do not.

deeper, more scattered neurons in the future white matter above the intermediate zone (Figs. 1, 2). These differences in cell position might relate to differences in their associated circuitry. Plotting the relative position of SPNs in the two groups within the subplate at different ages revealed that, at older ages, SPNs that receive layer 4 inputs were on average located more superficially (closer to the pia) than those that did not $(p<0.05)$ (Fig. 8). These results suggest that the subplate might contain a laminar organization with physiologically different subcircuits. However, the distributions showed a large degree of overlap, suggesting that in mouse there is some intermingling of these circuits.

\section{Discussion}

Our studies find that SPNs receive prominent excitatory and inhibitory inputs from the developing cortex. In most SPNs, the laminar source of these inputs is restricted to the deep layers in early life. Over development, a large ( $\sim 50 \%$ of total) subpopulation of SPNs receive inputs from superficial cortical layers including layer 4 . Thus, the subplate contains physiologically distinct subcircuits. We find that these circuits show some degree of spatial segregation, with upper SPNs being more likely to receive layer 4 inputs. This is consistent with recent observations that suggest a degree of compartmentalization, which is most apparent in primates and might reflect evolutionarily distinct origins (Montiel et al., 2011; Wang et al., 2011). SPNs receive major ascending inputs from the thalamus that they relay to layer 4. Our results here show that the activity of SPNs at later stages in development is also influenced by cortical activity, in particular that of layer 4. Figure 9 summarizes our current view of the development of subplate-associated circuits.

Previous studies have shown that SPNs show a large molecular and morphological diversity but that they are relatively homogenous based on their intrinsic electrical properties (Kanold and Luhmann, 2010). The observation of distinct subtypes of SPNs is consistent with reports of differences in synaptic properties between superficial and deep persistent SPNs in older animals (Torres-Reveron and Friedlander, 2007). Here, we show the existence of distinct input patterns to subpopulations of SPNs located at different locations within the subplate. Thus, the subplate is not a homogeneous structure, but contains multiple embedded circuits. While one of the main outputs of SPNs is cortical layer 4, SPNs also send projections back to the thalamus (McConnell et al., 1994). Thus, the multiple input patterns revealed here could relate to different functional output targets such as cortical layer 4 versus thalamus or differences between projection neurons and interneurons within the subplate. Our previous studies have shown that SPNs that project to layer 4 tend to be located superficially (Zhao et al., 2009); thus, it might be these neurons that receive feedback projections from layer 4 . The molecular identity and projection patterns of the deep SPNs remain to be elucidated. It is interesting to note that some deep SPNs (Fig. 3E) integrate inhibitory information from within the subplate over $\sim 1 \mathrm{~mm}$, which encompasses a large fraction of auditory cortex. While the functional role of such long-distance integration in the subplate is unclear, such long-distance interactions could be involved in the formation of organized cortical maps.

The fact that SPNs receive extensive cortical projections is consistent with the extensive neurites SPNs extend into the overlying cortical plate (Friauf et al., 1990; Friauf and Shatz, 1991; Torres-Reveron and Friedlander, 2007; Piñon et al., 2009; Zhao et al., 2009; Hoerder-Suabedissen and Molnár, 2011). While some of these are undoubtedly axons as SPNs excite cortical neurons (Zhao et al., 2009), our results here suggest that others are dendrites.

In young mice, we did not observe many SPNs that receive inputs from the superficial cortical plate. This can be due to the fact that cells in the cortical plate are immature and potentially might not express sufficient glutamate receptors, which would prevent them from being activated by LSPS. However, recordings from layer 4 neurons coupled with LSPS show that at young ages the majority of layer 4 neurons have glutamatergic receptors since spikes can be evoked (Fig. 2). Our results here suggest that projections from superficial neurons to SPNs have not formed or that projections might be anatomically present but are not func- 
tionally strong enough to be revealed with LSPS. Since a large fraction SPNs die during the developmental period (Kanold and Luhmann, 2010), the relative increase of SPNs could reflect the disappearance of one cell population or an increase in connectivity between the cortex and SPNs. The small numbers of SPNs receiving layer 4 input at young ages and the developmental extension of SPN neurites into the cortical plate (Piñon et al., 2009) are consistent with the latter scenario.

The columnar restriction of cortical inputs to SPNs is consistent with previous studies of the distribution of neurites of a selected subpopulation of SPNs expressing eGFP under the Golli promoter [Golli-tau-eGFP (GTE)] in barrel cortex (Piñon et al., 2009). There, SPN neurites were initially associated with the developing barrel patterns in S1 (Piñon et al., 2009). Interestingly, while neurites initially were associated with thalamic afferents, by P10 neurites and thalamic afferents segregated into barrels and septa (Piñon et al., 2009). Thus, if some of the neurites in the GTE mouse are dendrites, this suggests that feedback projections via SPNs can carry very different information that can get integrated with thalamic information within the SPN.

While the fractional input provides a way to compare the strength of inputs in different laminas, it does overestimate the contributions from areas away from the cell body. This is because stimulation close to the cell body in the subplate results in a large direct response, which masks EPSCs. Thus, our estimate of the total EPSC originating in the subplate might be an underestimate. However, the finding that more SPNs receive excitatory inputs from superficial layers over development is unaffected by this technical complication because direct responses are present in all cells at all ages.

In a few SPNs, we observed EPSCs and IPSCs after stimulation of layer 1 (Fig. 3). It has been reported that layer 1 might receive nonprimary thalamic input (Cruikshank et al., 2002). Thus, via this circuit, SPNs might receive both primary and nonprimary thalamic inputs. However, an alternative possibility is that the stimulation sites in layer 1 that give rise to EPSCs in SPNs are actually activating apical dendrites of layer $2 / 3$ or displaced layer $2 / 3$ neurons.

The amplitude of layer 4 inputs to SPNs is larger than minimal thalamic evoked EPSCs ( $15-20 \mathrm{pA})$ (Zhao et al., 2009), but much smaller than maximal thalamic input at older ages, which ranges over 120-180 pA (Zhao et al., 2009). This might reflect genuine differences in synaptic conductances or different amounts of dendritic attenuation since cortical inputs are likely to synapse on apical dendrites of SPNs (Fig. 1C). Nevertheless, since input resistances of SPNs are in the range of $280-550 \mathrm{M} \Omega$ during the studied time range (Zhao et al., 2009), the cortical inputs should be able to depolarize SPNs by $\sim 10-20 \mathrm{mV}$, and thus are able to significantly alter SPN excitability. SPNs also show inhibitory inputs from the cortical plate, but in most SPNs these inputs arose from deeper layers than the excitatory feedback input. These results suggest that the predominant effect of layer 4 feedback is excitatory.

What roles could layer 4 projections to SPNs play? Since SPNs project to layer 4 (Friauf et al., 1990; Zhao et al., 2009), excitatory feedback from layer 4 can aid in amplifying incoming activity. Thus, in early development, when thalamic axons are immature, this circuit might aid in the amplification of thalamic activity (Kanold and Luhmann, 2010). In addition, the excitatory feedback from layer 4 sets up an excitatory-excitatory circuit. Such a microcircuit might be involved in oscillatory activity. Changing patterns of oscillatory activity have been observed in the developing cortex and large changes are seen after P6 in rat (Yang et al.,
2009). We hypothesize that the changing microcircuits in the subplate are positioned to generate or modulate such oscillations. Such a role of SPNs is supported by recent lesion studies that show that removal of SPNs prevents the occurrence of spindle burst activity in the cortex (Tolner et al., 2009, 2012). Our data indicate that the excitatory feedback becomes more prominent with ear opening and the onset of low-threshold hearing (Fig. 5). In the visual system, eye opening is correlated with changes in the pattern of oscillatory activity in the cortex (Colonnese et al., 2010). The change in oscillatory activity over development might be due to the changes in cortical circuitry described here. Recent work indicated that sensory activity can influence the patterning of SPN neurites (Piñon et al., 2009). Thus, together these observations raise the possibility that the emergence of feedback from layer 4 might be caused by developmental changes in sensory evoked activity, in the case of the auditory system changes in cortical activity due to ear opening.

Previous studies of the functional role of SPNs relied on lesions or developmental insults that likely disrupted most if not all SPN circuits (Ghosh and Shatz, 1992; Kanold et al., 2003; Failor et al., 2006, 2010; Kanold and Shatz, 2006). The existence of distinct microcircuits in the subplate that might be associated with distinct molecular markers such as CTGF (Hoerder-Suabedissen et al., 2009; Osheroff and Hatten, 2009) will enable a more detailed dissection of the functional role of these separate circuits.

Our results show that SPNs are tightly embedded in the developing thalamocortical and intracortical circuit. SPNs receive and relay thalamic information to the developing cortical layer 4 . At young ages, these feedforward excitatory connections enable SPNs to control activity levels and patterns in layer 4 that ultimately lead to the functional maturation of layer 4 . However, at older ages SPNs also receive extensive inputs from the cortical plate, including at ages when the thalamocortical synapse is functional. Thus, at later ages, SPNs are part of both a feedforward and feedback circuit. Since a large fraction of SPNs die during development, it is possible that the subset of SPNs that are engaged in these feedforward and feedback circuits survives until adulthood. Together, our data show that SPNs are not a simple relay of information but are in the position to perform complex integrative functions throughout the prenatal and neonatal period.

\section{References}

Brill J, Huguenard JR (2009) Robust short-latency perisomatic inhibition onto neocortical pyramidal cells detected by laser-scanning photostimulation. J Neurosci 29:7413-7423.

Colonnese MT, Kaminska A, Minlebaev M, Milh M, Bloem B, Lescure S, Moriette G, Chiron C, Ben-Ari Y, Khazipov R (2010) A conserved switch in sensory processing prepares developing neocortex for vision. Neuron 67:480-498.

Cruikshank SJ, Rose HJ, Metherate R (2002) Auditory thalamocortical synaptic transmission in vitro. J Neurophysiol 87:361-384.

de Villers-Sidani E, Chang EF, Bao S, Merzenich MM (2007) Critical period window for spectral tuning defined in the primary auditory cortex (A1) in the rat. J Neurosci 27:180-189.

Failor S, Nguyen V, Darcy DP, Cang J, Wendland MF, Stryker MP, McQuillen PS (2010) Neonatal cerebral hypoxia-ischemia impairs plasticity in rat visual cortex. J Neurosci 30:81-92.

Failor SW, Evans MM, Cang J, Stryker MP, McQuillen PS (2006) Impaired cortical plasticity after early hypoxia-ischemia. Soc Neurosci Abstr 32:717.14.

Finney EM, Stone JR, Shatz CJ (1998) Major glutamatergic projection from subplate into visual cortex during development. J Comp Neurol 398:105-118.

Friauf E, Shatz CJ (1991) Changing patterns of synaptic input to subplate and cortical plate during development of visual cortex. J Neurophysiol 66:2059-2071. 
Friauf E, McConnell SK, Shatz CJ (1990) Functional synaptic circuits in the subplate during fetal and early postnatal development of cat visual cortex. J Neurosci 10:2601-2613.

Ghosh A, Shatz CJ (1992) Involvement of subplate neurons in the formation of ocular dominance columns. Science 255:1441-1443.

Hanganu IL, Luhmann HJ (2004) Functional nicotinic acetylcholine receptors on subplate neurons in neonatal rat somatosensory cortex. J Neurophysiol 92:189-198.

Hanganu IL, Kilb W, Luhmann HJ (2002) Functional synaptic projections onto subplate neurons in neonatal rat somatosensory cortex. J Neurosci 22:7165-7176.

Heuer H, Christ S, Friedrichsen S, Brauer D, Winckler M, Bauer K, Raivich G (2003) Connective tissue growth factor: a novel marker of layer VII neurons in the rat cerebral cortex. Neuroscience 119:43-52.

Higashi S, Molnár Z, Kurotani T, Toyama K (2002) Prenatal development of neural excitation in rat thalamocortical projections studied by optical recording. Neuroscience 115:1231-1246.

Hoerder-Suabedissen A, Molnár Z (2012) The morphology of mouse subplate cells with identified projection targets changes with age. J Comp Neurol 520:174-185.

Hoerder-Suabedissen A, Wang WZ, Lee S, Davies KE, Goffinet AM, Rakić S, Parnavelas J, Reim K, Nicolić M, Paulsen O, Molnár Z (2009) Novel markers reveal subpopulations of subplate neurons in the murine cerebral cortex. Cereb Cortex 19:1738-1750.

Hooks BM, Hires SA, Zhang YX, Huber D, Petreanu L, Svoboda K, Shepherd GM (2011) Laminar analysis of excitatory local circuits in vibrissal motor and sensory cortical areas. PLoS Biol 9:e1000572.

Kanold PO, Luhmann HJ (2010) The subplate and early cortical circuits. Annu Rev Neurosci 33:23-48.

Kanold PO, Shatz CJ (2006) Subplate neurons regulate maturation of cortical inhibition and outcome of ocular dominance plasticity. Neuron 51:627-638.

Kanold PO, Kara P, Reid RC, Shatz CJ (2003) Role of subplate neurons in functional maturation of visual cortical columns. Science 301:521-525.

Kao JPY (2006) Caged molecules: principles and practical considerations. In: Current protocols in neuroscience (Gerfen C, Holmes A, Rogawski M, Sibley D, Skolnick P, Wray S, eds), Unit 6.20. Hoboken, NJ: Wiley.

Kostovic I, Rakic P (1980) Cytology and time of origin of interstitial neurons in the white matter in infant and adult human and monkey telencephalon. J Neurocytol 9:219-242.

McConnell SK, Ghosh A, Shatz CJ (1994) Subplate pioneers and the formation of descending connections from cerebral cortex. J Neurosci 14:1892-1907.

Molnár Z, Kurotani T, Higashi S, Yamamoto N, Toyama K (2003) Development of functional thalamocortical synapses studied with current source-density analysis in whole forebrain slices in the rat. Brain Res Bull 60:355-371.

Montiel JF, Wang WZ, Oeschger FM, Hoerder-Suabedissen A, Tung WL, García-Moreno F, Holm IE, Villalón A, Molnár Z (2011) Hypothesis on the dual origin of the mammalian subplate. Front Neuroanat 5:25.
Nikolenko V, Poskanzer KE, Yuste R (2007) Two-photon photostimulation and imaging of neural circuits. Nat Methods 4:943-950.

Osheroff H, Hatten ME (2009) Gene expression profiling of preplate neurons destined for the subplate: genes involved in transcription, axon extension, neurotransmitter regulation, steroid hormone signaling, and neuronal survival. Cereb Cortex 19 [Suppl 1]:i126-i134.

Piñon MC, Jethwa A, Jacobs E, Campagnoni A, Molnár Z (2009) Dynamic integration of subplate neurons into the cortical barrel field circuitry during postnatal development in the Golli-tau-eGFP (GTE) mouse. J Physiol 587:1903-1915.

Pujol R, Lavigne-Rebillard, Lenoir M (1997) Development of sensory and neural structures in the mammalian cochlea. In: Development of the auditory system (Rubel E, Popper A, Fay R, eds), pp 146-192. Berlin: Springer.

Ruebsamen R, Lippe W (1997) The development of cochlear function. In: Development of the auditory system (Rubel E, Popper A, Fay R, eds), pp 193-270. Berlin: Springer.

Shepherd GM, Pologruto TA, Svoboda K (2003) Circuit analysis of experience-dependent plasticity in the developing rat barrel cortex. Neuron 38:277-289.

Shepherd GM, Stepanyants A, Bureau I, Chklovskii D, Svoboda K (2005) Geometric and functional organization of cortical circuits. Nat Neurosci 8:782-790.

Shnerson A, Pujol R (1983) Development: anatomy, electrophysiology, and behavior. In: The auditory psychobiology of the mouse (Willot J, ed), pp 395-425. Springfield, IL: Charles C. Thomas.

Tolner E, Yukin A, Kaila K, Kanold PO (2009) Subplate neurons promote the development of patterned cortical activity in neonatal rat cortex. Soc Neurosci Abstr 35:128.9.

Tolner EA, Sheikh A, Yukin AY, Kaila K, Kanold PO (2012) Subplate neurons promote spindle bursts and thalamocortical patterning in the neonatal rat somatosensory cortex. J Neurosci 32:692-702.

Torres-Reveron J, Friedlander MJ (2007) Properties of persistent postnatal cortical subplate neurons. J Neurosci 27:9962-9974.

Tritsch NX, Rodríguez-Contreras A, Crins TT, Wang HC, Borst JG, Bergles DE (2010) Calcium action potentials in hair cells pattern auditory neuron activity before hearing onset. Nat Neurosci 13:1050-1052.

Wang WZ, Oeschger FM, Montiel JF, García-Moreno F, HoerderSuabedissen A, Krubitzer L, Ek CJ, Saunders NR, Reim K, Villalón A, Molnár Z (2011) Comparative aspects of subplate zone studied with gene expression in sauropsids and mammals. Cereb Cortex 21: 2187-2203.

Watakabe A, Ichinohe N, Ohsawa S, Hashikawa T, Komatsu Y, Rockland KS, Yamamori T (2007) Comparative analysis of layer-specific genes in mammalian neocortex. Cereb Cortex 17:1918-1933.

Yang JW, Hanganu-Opatz IL, Sun JJ, Luhmann HJ (2009) Three patterns of oscillatory activity differentially synchronize developing neocortical networks in vivo. J Neurosci 29:9011-9025.

Zhao C, Kao JP, Kanold PO (2009) Functional excitatory microcircuits in neonatal cortex connect thalamus and layer 4. J Neurosci 29:1547915488 . 